\title{
Reactions of Polyfluorobenzenes With Nucleophilic Reagents ${ }^{1}$
}

\author{
Leo A. Wall, Walter J. Pummer, James E. Fearn, and Joseph M. Antonucci
}

(June 10, 1963)

\begin{abstract}
Nucleophilic reactions of hexafluorobenzene and related polyfluorobenzenes were studied in detail. Reaction of hexafluorobenzene with hydroxides, alcoholates, aqueous amines, and organolithium compounds led to the substitution of one or more fluorine atoms. The structures of the products were determined, using near infrared and nuclear magnetic resonance spectra. Fluorine is replaced more readily than chlorine, bromine, iodine, or other groups. In the majority of the products in which two of the fluorines in hexafluorobenzene were replaced, the substituting groups were para to each other. However, depending on the reagents other orientation effects were noted. The reaction mechanisms were a function of reagents and conditions. The most prevalent mechanism is presumably the displacement of a fluoride anion by another anion, probably via the formation of transition complexes of different lifetimes. However, simple ionization or attack by neutral species may occur under some conditions. The diazotization and oxidation of pentafluoroaniline were also investigated.
\end{abstract}

\section{Introduction}

Hexafluorobenzene $[1,2,3,4]^{2}$ and other highly fluorinated benzene derivatives [5] have been synthesized in recent years by several methods. These substances are very inert chemically and extremely stable to heat and radiation $[6,7,81$. Hexafluorobenzene has not, to date, been reported to react with electrophilic reagents. Exceedingly drastic acidic conditions have been required to halogenate further tetrafluoro- and pentafluoro benzene $[9,10,11,12]$. Hexafluorobenzene will add chlorine $[1,2,4]$ and is readily attacked by free radicals [13].

Like hexachlorobenzene [14], hexafluorobenzene would be expected to react with bases. Strong nucleophilic reagents will displace fluorine from the aromatic ring. The strong inductive effect of the electronegative fluorine atoms should produce highly positive ring-carbon atoms. Unlike hexachlorobenzene, which is a high-melting solid poorly soluble in organic solvents, hexafluorobenzene is a liquid and is soluble in many solvents. Hence, the latter compound readily reacts with many bases in appropriate solvents. Under pressure at elevated temperatures, aqueous systems have also been used successfully with hexafluorobenzene.

The reactions of hexafluorobenzene and many of its monosubstituted derivatives with various nucleophilic reagents will be described in this paper. The chief reagents utilized were alkali-metal hydroxides, alcoholates, amines, and organolithium compounds. In general, these reagents replace fluorine in preference to bromine or iodine, for example, in reactions

${ }^{1}$ Based on research supported by the Bureau of Naval Weapons, Department of the Navy.

2 Figures in brackets indicate the literature references at the end of this paper with bromo- and iodo-pentafluorobenzene. This is in accord with previously observed results on nucleophilic reactions $[15,16]$. With 1 -chloro-3,4,5,6tetrafluorobenzene, the chlorine atom can, however, be replaced preferentially by a hydrogen atom [17]. The reaction is carried out with hydrogen over a palladium catalyst at $280^{\circ} \mathrm{C}$.

Many of the reactions studied are informative of the directional effects created by groups other than a fluorine atom in an otherwise completely fluorinated benzene ring. Very meager information of this nature has been available previous to this work. Nuclear magnetic-resonance spectroscopy was used as the primary method for determining molecular structure. Infrared spectra, particularly in the near-infrared, were also of assistance in determining structure, especially in the case of the ortho hydroxyl compounds.

\section{Reactions of Hexafluorobenzene}

\subsection{Reactions With Hydroxides}

It has been found that polyfluoroaromatic phenols can be prepared readily by the following type of reaction:

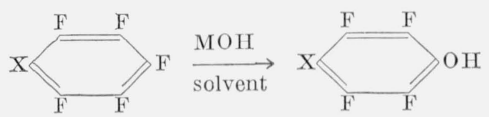

where $\mathrm{M}$ is any alkali metal and $\mathrm{X}$ is a fluorine atom or some other substituent group. The product is often the para isomer, but mixtures of isomers may occur. 
In our earlier work [18], pentafluorophenol was prepared by refluxing hexafluorobenzene with potassium hydroxide in pyridine, which was found to be an effective solvent for this reaction. In similar reactions with hexachlorobenzene, it has been suggested [14] that pyridine has a specific, catalytic effect. With ethanolic pyridine as solvent, the reaction gave pentafluorophenol and tetrafluorodihydroxybenzene together with small proportions of pentafluorophenetole and diethoxytetrafluorobenzene. Since the phenol reacts further only with difficulty due presumably to the repulsion of the negative ion by the phenoxy ion, it is likely that the disubstituted products result via reaction of the base with the phenetole and that many substituted ethoxy groups are then split by the basic medium to give the hydroxy derivatives. With tert-butyl alcohol as diluent, other workers have prepared pentafluorophenol from hexafluorobenzene [19].

In the present work, it was found that simple, aqueous systems are adequate for these nucleophilic reactions. Thus, we have prepared pentafluorophenol in high yield $(85 \%)$ by using aqueous potassium hydroxide at $175^{\circ} \mathrm{C}$ in a closed pressure-vessel. For the phenol preparation, our experience suggests water to be the preferred diluent, followed by tertbutyl alcohol. Tetrafluoro-p-cresol was prepared, using tert-butyl alcohol as diluent. With pyridine as solvent, black tars are undesired by-products; using pyridine, the compounds 2,3,5,6-tetrafluorophenol, 2-bromo-3,4,5,6-tetrafluorophenol, 4-bromo-2,3,5,6tetrafluorophenol, and 2,3,5,6-tetrafluoro-4-iodophenol have been prepared.

\subsection{Reactions With Alkoxides}

Another reaction of polyfluoroaromatics investigated was that with such alcoholates as sodium methoxide.

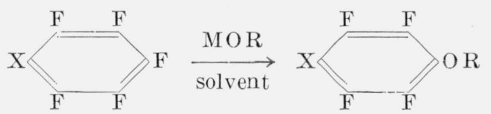

The reaction is usually carried out at reflux temperatures, to give good yields of ethers of the general type shown. With sodium methoxide in methanol, pentafluoroanisole was produced $[4,20]$, together with some of the tetrafluoro- $p$-dimethoxybenzene. With pyridine - methanol as the solvent [18], rapid reaction occurred to produce high yields $(70 \%)$ of pentafluoroanisole. The tetrafluoro- $p$-dimethoxybenzene has also been produced by other methods [21, 22], e.g., from the reaction of diazomethane with $2,3,5,6-$ tetrafluorohydroquinone. Synthesis by this route established that the second methoxyl group is in the para position to the first.

The pentafluoroanisole may be demethylated, with difficulty, by refluxing with 47-percent hydriodic acid [3], to give a 20-percent yield of pentafluorophenol. Demethylation is also relatively poor with hydrobromic acid [20]. However, when the pentafluoroanisole is heated with anhydrous aluminum chloride and the mixture is poured onto ice, a 58 percent yield of pentafluorophenol [20] is obtained.
Pentafluorophenol is extremely resistant to further substitution by nucleophilic reagents, probably because these reactions would require the attack of a negative species on the negative phenoxide ion. Ammonium 2,3,4,5,6-pentafluorophenoxide was the only product obtained from treatment of (a) pentafluorophenol, (b) sodium pentafluorophenoxide, or (c) ammonium pentafluorophenoxide with sodamide in liquid ammonia. Thus, the tetrafluorodihydroxybenzene [18] produced in the reaction with ethanolic potassium hydroxide in pyridine probably arises form the attack of a nucleophilic species on the phenetole. In another study [19], only the phenetole was produced in 48 percent yield, and 45 percent of the hexafluorobenzene was recovered after reaction with ethanolic potassium hydroxide for $30 \mathrm{~min}$ at $120^{\circ} \mathrm{C}$. In accord with this result is the observation made in this work that the treatment of pentafluoroanisole with aqueous ammonia results in the isolation of ammonium pentafluorophenoxide. With hydrazine hydrate in ethanol, hydrazinium pentafluorophenoxide is obtained [20]. However, benzyl pentafluorophenyl ether is not readily split by nitrogen bases, and a fair yield (13\%) of benzyloxytetrafluoroaniline was obtained by treating it with aqueous ammonia.

In this work, bromo- and iodo-pentafluorobenzene were converted to the corresponding anisoles in which a fluorine atom is replaced by a methoxyl group. Reaction of sodium benzyloxide (in excess benzyl alcohol or in tert-butyl alcohol) with hexafluorobenzene gave benzyl pentafluorophenyl ether. A higher yield of purer material was obtained when the latter solvent was used, since benzyl alcohol is difficult to remove from the product.

Anhydrous sodium phenoxide was found to react rapidly with hexafluorobenzene at room temperature in $N, N$-dimethylformamide, giving $2,3,4,5,6$-pentafluorophenyl phenyl ether. This compound was also prepared by treating potassium pentafluorophenoxide with bromobenzene, using activated copper as a catalyst. This reaction failed to give the perfluorophenyl ether when bromopentafluorobenzene was used instead of bromobenzene; the starting materials were recovered.

Perfluorophenyl ether was, however, prepared, although in relatively low yield, by reacting potassium pentafluorophenoxide with hexafluorobenzene in $N, N$-dimethylformamide. This reaction was more sluggish than that involved in the preparation of the pentafluorophenyl phenyl ether and required refluxing. This difference may be partially explained by the poorer nucleophilic nature of the potassium pentafluorophenoxide as compared to the sodium phenoxide.

By the reaction of sodium ethoxide in ethanol with pentafluoro- $N, N$-dimethylaniline, 4-ethoxy-2,3,5,6tetrafluoro- $N, N$-dimethylaniline was synthesized. No pyridine was used in this reaction, since phenol formation is likely to occur under these conditions.

\subsection{Reactions With Amines}

Hexafluorobenzene has been shown to undergo reaction with sodamide at $-70{ }^{\circ} \mathrm{C}$ in liquid ammonia, 
to give 2,3,4,5,6-pentafluoroaniline [23]. In ether, reaction with sodamide did not produce pentafluoroaniline; a solid which slowly sublimed at $85^{\circ} \mathrm{C}$ was obtained [24]. The preferred reaction for replacing fluorine atoms with amino groups is treatment with aqueous amines [14].

In this investigation, the reaction of aqueous amines with polyfluoroaromatic compounds has been extensively explored.

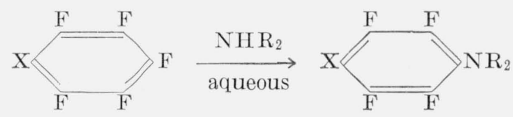

Our result differed in certain respects from those obtained when aqueous ethanolic amine solutions were used [25]. The reactions were carried out at 160 to $230{ }^{\circ} \mathrm{C}$ in a steel pressure-vessel. Control of temperature and time of the reaction is critical since, at low temperature, the reaction does not occur and, at higher temperatures, unidentified tars are produced.

In this investigation, bromo- and iodo-tetrafluoroanilines, pentafluoroaniline, and various polyfluoromethylanilines were prepared by the aqueous amine process. Although (benzyloxy)tetrafluoroaniline was formed by the reaction of benzyl pentafluorophenyl ether with aqueous ammonia, only ammonium pentafluorophenoxide was produced from $2,3,4,5,6$-pentafluoroanisole. It was found, however, that, in the reaction of pentafluoroanisole with sodamide in liquid ammonia, normal replacement of a fluorine atom occurred (instead of cleavage of the methoxy group) to give 2,3,5,6-tetrafluoroanisidine. Since the fluoroanisidine formed is amphoteric, such products as the di- and tri-anisylamines were also formed.

\section{a. Diazotization of Pentafluoroaniline}

The versatility of the "diazo reactions," i.e., the Sandmever reactions, is well known in aromatic chemistry as they provide a means of preparing a variety of compounds not obtainable by other methods. It was of interest, therefore, to determine the usefulness of the Sandmeyer reaction for preparing pentafluoroiodobenzene and bromopentafluorobenzene.

Pentafluoroaniline is weakly basic, reacts slowly with nitrous acid, and dissolves only in concentrated strong acids [23]. On dilution with water, however, the free base is regenerated; this demonstrates that the salt of the amine hydrolyzes readily, which is an important factor in the diazotization process in aqueous solutions. Diazotization of pentafluoroaniline in anhydrous hydrogen fluoride and concentrated sulfuric acid has been studied by us. It can be oxidized to give a variety of products; pentafluoronitrobenzene [26], pentafluoronitrosobenzene [27], and decafluoroazoxybenzene are described herein.

The diazotization of pentafluoroaniline to yield deca$\mathrm{H}$

fluorodiazoaminobenzene, $\quad \mathrm{C}_{6} \mathrm{~F}_{5}-\mathrm{N}=\mathrm{N}-\mathrm{N}-\mathrm{C}_{6} \mathrm{~F}_{5}$, has been reported previously [23], but no experimental details were given. We have found that this compound is obtained when concentrated hydrobromic acid $(48 \%)$ is used as the reaction medium; its formation may be attributable to the slow diazotization of the amine (rather than to a too low concentration of acid, which is sometimes the cause for this coupling reaction [28]). The reaction also appears to be temperature-dependent, because the rate of formation of the diazoaminobenzene is much faster at $10{ }^{\circ} \mathrm{C}$ than at $-10{ }^{\circ} \mathrm{C}$. Allowing the latter reaction mixture to warm to $5{ }^{\circ} \mathrm{C}$ causes the compound to be precipitated.

Decafluorodiazoaminobenzene can be decomposed in warm hydrobromic acid solution in the presence of cuprous bromide. The products obtained are bromopentafluorobenzene $(5.4 \%)$ and a mixture of dibromotetrafluorobenzenes $(11.7 \%)$, presumably the $o$ - and $p$-isomers. The isolation of the latter compounds suggests that the diazo group exerts a considerable influence on the ortho and para fluorine atoms, making possible the replacement with bromide anion. This replacement reaction apparently occurs prior to the decomposition of the diazo group, because bromopentafluorobenzene is stable to concentrated mineral acids $[12,29]$. Similar replacement reactions have been observed [23] when the diazotizations were performed under neutral or alkaline conditions.

In such nonaqueous solvents as concentrated sulfuric acid, the diazotization of pentafluoroaniline is extremely slow, even at $25{ }^{\circ} \mathrm{C}$. The addition of glacial acetic acid tends to hasten the reaction slightly. The deep-red color appears only after 24 hrs. Deamination with hypophosphorous acid yields a product believed to be a mixture of pentafluorobenzene and tetrafluorobenzene. These compounds are difficult to separate, even by vapor-phase chromatography. Dilution of the diazo solution with water reprecipitates the yellow diazoaminobenzene, as mentioned previously.

The use of anhydrous liquid hydrogen fluoride as the solvent and reaction medium resulted in a decided improvement in the yield of product. Liquid hydrogen fluoride has found moderate use in diazonium reactions, even though fluorobenzene can be prepared in 86 percent yield from aniline in this medium [30]. We have found that the diazotization of pentafluoroaniline is fairly rapid, even at $-25{ }^{\circ} \mathrm{C}$. Pentafluoroidobenzene and bromopentafluorobenzene can be prepared in 50 percent and 35 percent yield, respectively, by normal Sandmeyer reagents (potassium iodide-or potassium bromidecuprous bromide mixture). No other replacement products were observed during these reactions.

Another synthesis involving diazotization was the attempted preparation of the fluorinated diazo ether $\mathrm{C}_{6} \mathrm{~F}_{5}-\mathrm{N}=\mathrm{N}-\mathrm{O}-\mathrm{C}_{6} \mathrm{~F}_{5}$. Thermal decomposition of this material could possibly lead to the hitherto unknown perfluorophenyl ether. The product isloted from the reaction of lithium pentafluorophenoxide and the diazotized amine could be extracted into methylene chloride. However, after partial removal of the solvent, the material detonated violently. The instability of some substituted aroyl diazo ethers is well known [28], and our results 
indicate that this fluorodiazo ether may also be unstable.

The Sandmeyer nitrile synthesis failed to give any identified products with diazotized pentafluoroaniline. It has been previously observed $[12,29]$ that cyanide ion has an adverse effect on aromatic fluorine compounds, causing multiple replacement with subsequent decomposition, followed by carbonization. These results indicate that the diazonium reaction may have only limited usefulness in aromatic fluorocarbon chemistry.

\section{b. Oxidation of Pentafluoroaniline}

The oxidation of aromatic amines to nitro derivatives has been accomplished previously by using peroxy acids $[31,32,33]$. This procedure can also be applied to pentafluoroaniline $[26,27]$, and both pentafluoronitrobenzene [26] and pentafluoronitrosobenzene [27] have been synthesized. For oxidation of pentafluoroaniline, we used the conditions described by Holmes [34]. However, instead of the expected pentafluoronitrosobenzene, only decafluoroazoxybenzene was isolated as the final product, regardless of whether the reaction was allowed to proceed at room temperature or if heat was applied; when heat $\left(60\right.$ to $\left.75{ }^{\circ} \mathrm{C}\right)$ was used, the reaction proceeded mush faster. Presumably, from the color changes observed during the course of the reaction, it should be feasible to isolate the pentafluoronitrosobenzene by quenching the reaction at the green-color stage. Pentafluoronitrosobenzene has been shown [27] to exist as a monomer. However, one attempt to terminate the reaction at this stage led only to tarry products.

The formation of the decafluoroazoxybenzene as the main product seems to indicate the relative ease of the oxidation of decafluoroazobenzene (orange stage) with excess hydrogen peroxide. This reaction also occurs in the hydrocarbon series. The fact that no pentaflouronitrobenzene was obtained indicates that oxidation is not the sole reaction. In view of the rapid oxidation occurring when 90 percent peroxide was used [26], it would appear that, at the nitroso stage in our work, coupling with unused pentafluoroaniline predominates over further oxidation of the nitroso derivative. These reactions are illustrated as follows:
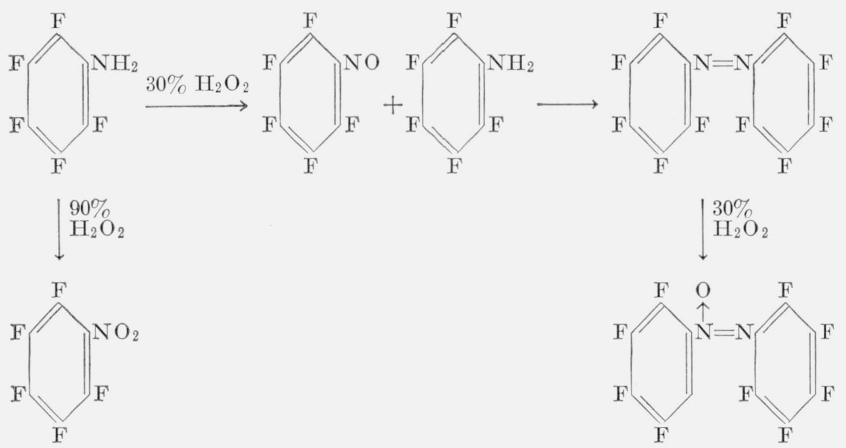

The decafluoroazobenzene can be readily prepared from the azoxy compound by reduction with zinc and ammonium chloride in methanol. Traces of pen- tafluoroaniline and an additional compound (which may be the decafluorohydroazobenzene) were also obtained.

\subsection{Reactions With Organolithium Compounds}

In some earlier work from this laboratory, it was found that the Grignard reagent, methylmagnesium bromide, reacts with hexafluorobenzene to produce 2,3,4,5,6-pentafluorotoluene. Under the conditions described [18], the yield was low (3\%). Alkyl-, alkenyl-, and aryl-lithium reacted readily to give the corresponding substituted pentafluorobenzenes.

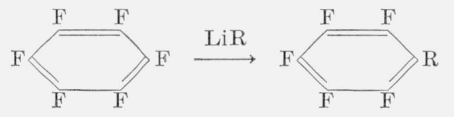

Methyl- and butyl-lithium reacted readily [35], as well as phenyl-, vinyl-, and isopropenyl-lithium [36].

The reactions are rapid, and some di- [35, 36, 37], as well as tri- $[26,35]$, substituted products were obtained. The preferred method of reaction is usually the addition of a solution of the lithium reagent to a solution of the aromatic fluorocarbon. Even so, disubstitution readily occurs. In the methyllithium reaction, 2,3,5,6-tetrafluoroxylene was identified [37], indicating that the second displacement of fluorine is para to the first. The yields of the pentafluorotoluene and 2,3,4,5,6-pentafluorobiphenyl reach 70 percent or higher. Butyllithium has so far given lower yields, but this reagent has been studied relatively little. The alkenyllithium reactions gave yields of the order of 30 to 50 percent, but this is the result of the further reaction of the products to give polymeric solids [38]. The lithium reagents themselves are good catalysts for the anionic polymerization of olefins.

2,3,4,5,6-Pentafluorotoluene can be halogenated to the corresponding benzyl chloride and bromide [37]. With chlorinating reagents, ring addition of halogen interferes with the production of the benzotrichloride [39]. The benzyl halides are versatile reagents for further synthesis of fluoroaryl compounds [37, 39].

In the course of our investigations of the reactions of hexafluorobenzene with organolithium compounds, it was decided to study the reaction of the simplest nucleophile, the hydride ion, with hexafluorobenzene. It was found that hexafluorobenzene does indeed undergo nucleophilic attack by the hydride ion to give pentafluorobenzene as the major product (together with traces of tetrafluorobenzene). The extent of conversion seems to depend on the proportion of lithium aluminum hydride used.

The reaction apparently occurs only when lithium aluminum hydride is the source of the hydride ions. When lithium hydride was employed, no formation of pentafluorobenzene or other products was observed. However, if the lithium hydride was used together with a small quantity of lithium aluminum hydride as a hydrogen carrier [40], a 25-percent conversion to pentafluorobenzene was observed. Again, a very small proportion of disubstitution product was formed; this product was presumably 1,2,4,5-tetrafluorobenzene, since this compound has 
been reported to be formed [40] when pentafluorobenzene is treated with lithium aluminum hydride. In the latter case, in addition to the tetrafluorobenzene, a high-boiling liquid is obtained in about 5 -percent yield; the residue distils in the range of 80 to $20{ }^{\circ} \mathrm{C}$ at $5 \mathrm{~mm}$. (Direct replacement of fluorine atoms by hydrogen atoms on the aromatic nucleus can also be accomplished by the use of hydrogen over platinum-on-carbon at $300{ }^{\circ} \mathrm{C}$ [17].)

\subsection{Mechanism of Reaction}

In this work, the reactivity of hexafluorobenzene toward a great variety of nucleophilic reagents has been explored. No specific investigations of the mechanistic features of the reactions involved were carried out, and the details of the various mechanisms must be left for future studies. No single mechanism is likely to be adequate for all of the reactions reported. In fact, the variety of conditions and reagents examined render it extremely likely that a variety of mechanisms is involved in the reactions reported.

The mechanisms of the nucleophilic reactions of aromatic systems have been reviewed [42, 43, 44]. The effects of solvents on these reactions has, apparently, not yet been investigated in great detail. However, review [45] of the effects of solvation on the properties of anions in dipolar, aprotic solvents discusses many aspects of nucleophilic mechanisms and the role of the solvent in them. In our work a variety of solvents, protic and aprotic, were utilized.

Although no specific kinetic work was carried out, the large amount and variety of results obtained allow certain mechanistic concepts to be developed. It seems evident, for instance, that a benzyne intermediate is not involved in our reported reactions of hexafluorobenzene in which a monosubstituted pentafluorobenzene is produced (even when it is treated with sodamide in liquid ammonia). This reaction of hexafluorobenzene is relatively efficient and is encumbered chiefly by the reactivity of the aniline product, which can react (via the anilide ion) to afford such products as perfluorodiphenylamine [25]. Tetrafluorobenzyne has, however, been reported [46] as a decomposition product of lithium pentafluorophenyl prepared from lithium amalgam and bromopentafluorobenzene. It is apparently stable but quite reactive. It would seem that benzyne formation in alkali-metal amide reactions in liquid ammonia is a secondary aspect of these nucleophilic reactions, although it explains various isomerizations observed in systems containing aromatic hydrogen atoms.

The reactions of hexafluorobenzene with alkalimetal hydroxides and alkoxides, sodamide, and organolithium compounds presumably involve attack on the hexafluorobenzene by negative ions, and it seems evident that, as a rule, unimolecular ionization of the hexafluorobenzene does not occur. The preparation of pentafluorophenol by treating potassium hydroxide in tert-butyl alcohol with hexafluorobenzene, and the preparation of benzyloxypentafluoro- benzene using sodium benzylate in tert-butyl alcohol, indicate this; otherwise, it would be expected that tert-butyl pentafluorophenyl ether would have been produced. The use of alcoholic and aqueous amine solutions to produce various anilines in which moieties from the solvents are not part of the final products also indicates absence of any appreciable ionization. Furthermore, no reaction occurs between hexafluorobenzene and pure water up to 300 ${ }^{\circ} \mathrm{C}$, the highest temperature studied. At the higher temperature, addition of a drop of pyridine led to the rapid formation of tarry material, which gave a phenolic odor on acidification; the quantities of tar or char were approximately proportional to the pyridine added. The effect of aqueous potassium cyanide on hexafluorobenzene was also studied in the temperature range of 150 to $250{ }^{\circ} \mathrm{C}$. Only at $250{ }^{\circ} \mathrm{C}$ did a reaction occur. However, no identified products were obtained, but only intractable chars. The reaction was violent and was essentially a contained explosion. Extensive damage to the pressure vessel occurred in one instance, although without violent fragmentation of the vessel. A similar study of the effect of aqueous sodium carbonate showed no reaction up to $250{ }^{\circ} \mathrm{C}$, at which temperature, there were formed extensive tars which again gave a phenolic odor on acidification.

Specific complexes probably play an important role in the reported reactions of hexafluorobenzene. It has been observed that a 1:1 molar mixture of benzene, $\mathrm{mp} 5.4^{\circ} \mathrm{C}$, and hexafluorobenzene, $\mathrm{mp} 5.0$ ${ }^{\circ} \mathrm{C}$, forms a solid melting at $23.7{ }^{\circ} \mathrm{C}$ [47], which demonstrates the formation of a molecular complex between the two species. Freezing-point data indicate complex-formation between hexafluorobenzene and fluorobenzene also, but not between pentafluorobenzene and benzene [47]. These complexes are presumably similar to the charge-transfer complexes known to form between picric acid and certain aromatic compounds. Benzene and 1,3,5-trinitrobenzene, for instance, form a complex [48] which is certainly of a similar character. With more electronreleasing substituents on a benzene nucleus, a more stable complex should form, as with hexafluorobenzene. In the case of mesitylene and hexafluorobenzene, a complex melting at $24{ }^{\circ} \mathrm{C}$ has been observed; one melting at $56{ }^{\circ} \mathrm{C}$ is formed between hexafluorobenzene and 2-methylnaphthalene [47]. These observations point to a specific complex-formation as an explanation of the role of pyridine in enhancing the reactivity of hexafluorobenzene in the reactions reported here. In aqueous systems, pyridine may be effecting simple ionization of the hexafluorobenzene.

In the reactions of hexafluorobenzene with ammonia or primary and secondary amines, the mechanism is probably one in which the un-ionized amine directly attacks the fluoroaromatic molecule:

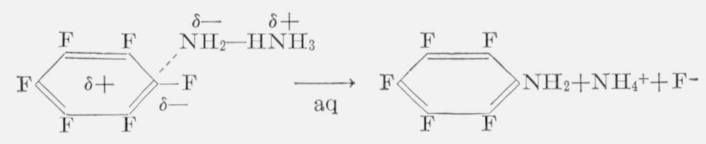

Whether the second ammonia molecule is nearby or 
not appears to be unimportant; a transitory hydronium ion would be equally effective.

The action of neutral water species, although obviously much less effective than nitrogen compounds, is likely to be involved to some extent in the formation, for instance, of pentafluorophenol by the reaction of aqueous alkali hydroxides with hexafluorobenzene.

By analogy with the formation of such complexes as that [42] formed by the addition of a methoxide ion to 2,4,6-trinitrophenetole, it is likely that hexafluorobenzene in its reactions with the amide, hydroxy, alkoxy, and alkyl anions forms a relatively stable, transition state.

A somewhat startling and puzzling facet of the reactions of fluorobenzene derivatives is the splitting of the alkoxypentafluorobenzenes by aqueous ammonia or hydrazine [20] and, presumably, by alcoholic potassium hydroxide [18]. In the hydrazine reaction, the hydrazine salt of pentafluorophenol is obtained. This reaction has been postulated to occur via a splitting of the methyl-oxygen link [20], presumably, as shown in the following series of reactions.

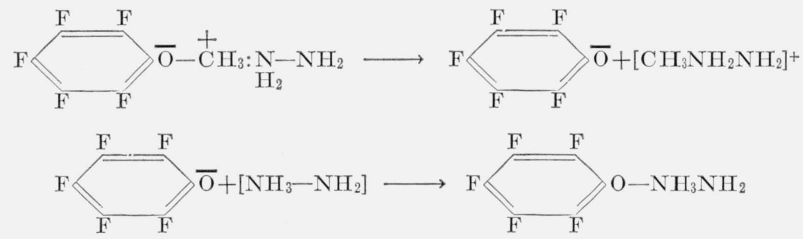

This splitting has only been observed in the presence of water, and is not necessarily related to the basicity of the medium; it does not occur in the liquid ammonia-sodamide reaction, where a fluorine atom is replaced without splitting of the ether link. However, sodamide in liquid ammonia does split anisole. Thus, it is possible that the split occurs at the pentafluorophenyl-oxygen link, and that water is the key reagent. For the reaction to occur at the phenyl-oxygen link, one can logically postulate either an attack by the hydroxide ion, which implies that the ring carbon atom at this site is highly positive, or some direct reaction at this carbon atom with a neutral water molecule. The former implies easier ionization of the phenyloxygen link, and not necessarily an enhancement of the positive character of the ring carbon atom linked to the oxygen atom. It is conceivable that the methyl-oxygen link is quite covalent. Since water was present in all of the reactions in which cleavage of the ether moiety was observed, it seems feasible that simple hydrolysis may have occurred. However, distilled water failed to react with pentafluoroanisole under conditions identical with those under which aqueous amine bases produced cleavage. Thus, the role played by the water molecules may simply be as a co-catalyst with the base, to aid polarization of the ether linkage, in much the manner suggested previously for pyridine. Determination of the bond actually ruptured would require experiments with $\mathrm{H}_{2} \mathrm{O}^{18}$.

\section{Identification of Isomers}

The orientation of the isomers of most of the difunctional derivatives of hexafluorobenzene of the general formula, $\mathrm{C}_{6} \mathrm{~F}_{4} X Y$, where $X$ and $Y$ may be similar or dissimilar groups (but not a fluorine atom), has been determined by nuclear magneticresonance spectroscopy. Direct syntheses of these derivatives for comparison and identification purposes by alternative routes has been difficult, although this has been accomplished in several cases $[22,25]$. Infrared and ultraviolet spectroscopy have so far not found extensive use in identifying structural isomers of aromatic fluorocarbons. In a series of articles, Baker $[49,50]$ has described the vibrational spectra of $o$-halophenols in the nearinfrared region; these compounds show a doubling of the hydroxyl stretching frequencies at low concentrations. This doubling of the hydroxyl band is absent for para-substituted halophenols and presumably for meta-substituted halophenols, although slight shifts of hydroxyl frequency do occur. Therefore, this method appeared to be a useful supplement to nuclear magnetic-resonance in distinguishing between the isomeric phenols obtained from the reaction of various pentafluorohalobenzenes with alkali hydroxides. The procedure appears to be specific for $o$-halophenols, in particular when the halogen atoms differ, because of the two types of hydrogen bonding that can occur. For example, the structures applied in our case to the 2-bromo-3, 4,5,6-tetrafluorophenol are:

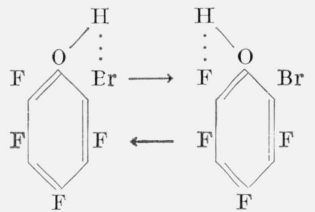

In figure $1-\mathbf{A}$, this compound exhibits the split hydroxyl band indicative of different halogen atoms, surrounding the hydroxyl group at the ortho positions. In this case, the $\Delta \nu \mathrm{OH}$ for fluorine is at $2.80 \mu$, and the $\Delta \nu \mathrm{OH}$ for bromine at $2.84 \mu$ is in agreement with values shown by Baker [50]. This doublet is absent from, and only a lone hydroxy band appears in, the spectrum of 4 -bromo-2,3,5,6tetrafluorophenol at $2.80 \mu$. However, since the 3-bromo-2,4,5,6-tetrafluorophenol is unknown at present, it is imposssible to ascertain whether or not the (large) bromine atom at this position would cause some steric effect on the ortho fluorine atom so as to change the electron environment whereby a doublet may be obtained instead of the single band. However, as expected, various tetrafluorophenols having substituents in the para position show the single hydroxyl band at 2.80 to $2.81 \mu$, similar to those shown in figure $1-\mathrm{B}$. Some of these phenols are: 2,3,5,6-tetrafluorophenol, 2,3,4,5,6pentafluorophenol, 2,3,5,6-tetrafluoro-p-cresol, and 2,3,5,6-tetrafluoro-4-iodophenol. By reverse reasoning, therefore, the absence of the split hydroxyl band in the near-infrared region in these simple 


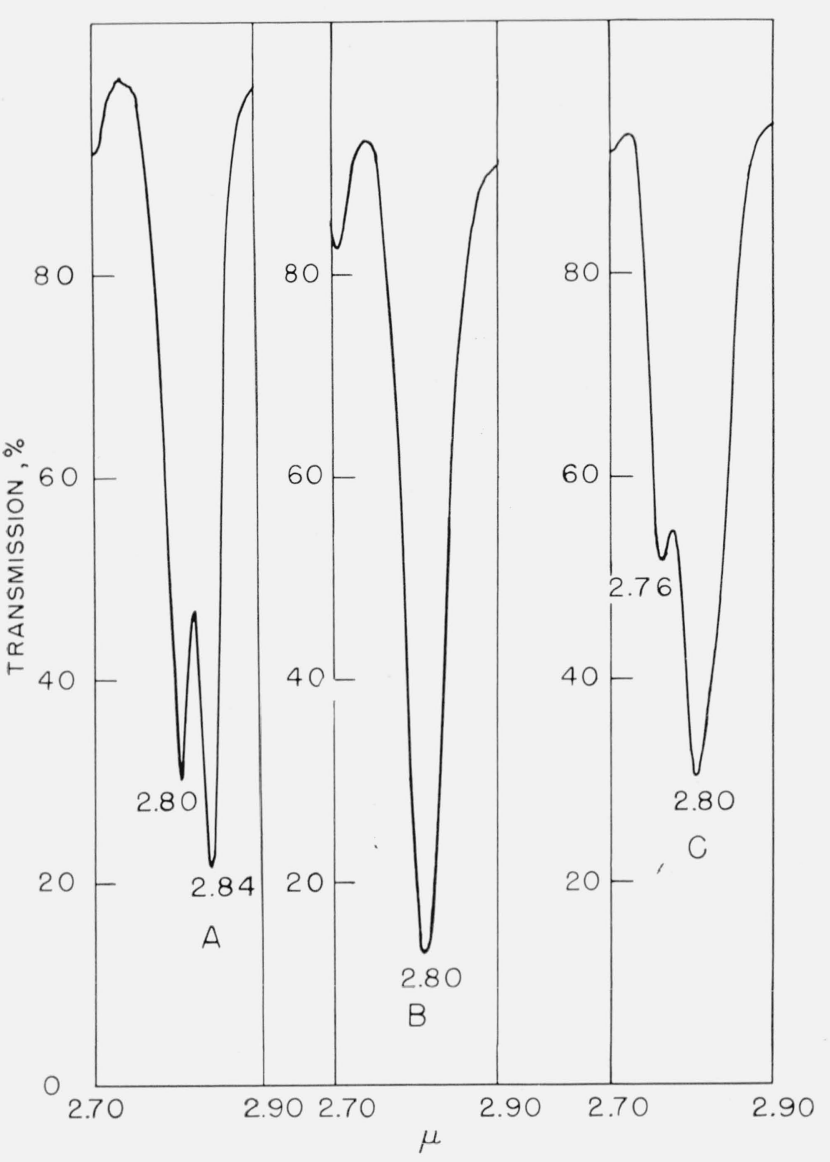

Figure 1. The OH spectra of halophenols in carbon tetrachloride.

A. 2-Bromo-3,4,5,6-tetrafluorophenol;

B. 2-Chloro-trifluoro- $\alpha, \alpha, \alpha$-trifluoro- $p$-cresol;

C. 2-Chloro-trifluoro- $\alpha, \alpha, \alpha$-trifluoro-o-cresol.

halophenols is indicative that groups other than fluorine atoms are not ortho to each other. These compounds were further confirmed by nuclear magnetic-resonance spectroscopy to be para isomers.

An attempt to extend this method of distinguishing. isomeric phenols to a more complex molecule has met with partial success. For example, in figures 1-B and $1-\mathrm{C}$ are shown the spectra of two phenolic products obtained from the reaction of 2-chloro-3,4,5, 6 -tetrafluoro- $\alpha, \alpha, \alpha$-trifluorotoluene with potassium hydroxide in pyridine. Since, in figure 1-B, only the single hydroxyl band appears, it is obvious that the hydroxyl group is located in either the 4 or the 5 position. From other considerations, the trifluoromethyl group would be expected to exert a greater directive effect than the chlorine atom. In this case, then, the hydroxyl group is probably located para to the trifluoromethyl group. However, in figure 1-C, the hydroxyl group would appear to be ortho to the trifluoromethyl group, since the $\Delta \nu \mathrm{OH}$ for chlorine would be expected to be at a higher wavelength (at $2.82 \mu$ ). The band at $2.76 \mu$ may in this instance again arise from the change in frequency of the hydroxyl group because of the two types of hydrogen bonding possible, for example, a five-membered bridge (I) versus a six-membered

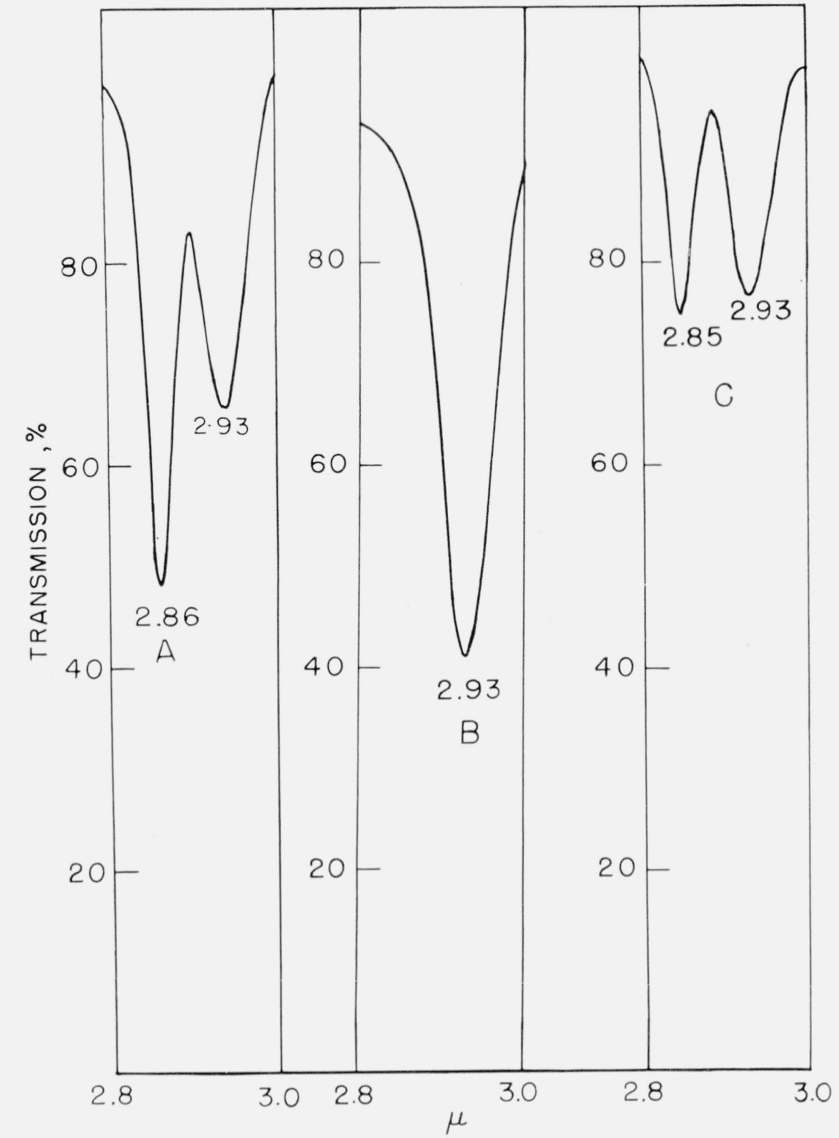

Figure 2. The $\mathrm{NH}_{2}$ and $\mathrm{NH}$ spectra of haloanilines in carbon tetrachloride.

A. 2,3,4,5,6-Pentafluoroaniline;

B. 2,3,5,6-Tetrafluoro-1,4-bis ( $N$-methylamino) benzene;

C. 2-Chloro-trifluoro- $\alpha, \alpha, \alpha$-trifiuoro- $p$-toluidine.

bridge (II), as shown below:

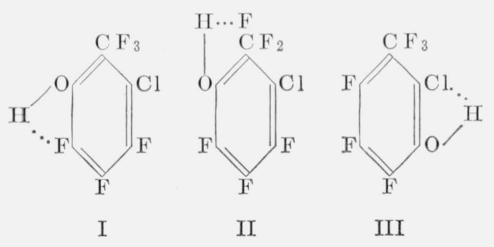

It could be argued that this band may be attributable to a free (trans) hydroxyl group, such as occurs with other ortho-monohalogenated $(\mathrm{Cl}, \mathrm{Br}, \mathrm{I})$ phenols, but this doubling has not been observed in o-fluorophenol [49]. Similarly, the vapor infrared spectrum of pentafluorophenol has a strong band at $2.75 \mu$ attributed to a free hydroxyl group [19], but, in a nonpolar solvent such as carbon tetrachloride, the band is shifted to a higher wavelength (at $2.80 \mu$ ). Therefore, the band observed in figure 1-C at $2.76 \mu$ is probably associated with the o-trifluoromethyl group, and not with a free hydroxyl group or an $o$-chlorine atom. On this basis, then, tentative identification of 3-chloro-4,5,6-trifluoro-2-( $\alpha, \alpha, \alpha$-tri fluoromethyl) phenol has been made, and the com- 
pound has been assigned the structures shown in I and II.

Attempts to apply this "ortho effect" to other derivatives of hexafluorobenzene, particularly to the aniline compounds, has been fruitless. For example, in the near-infrared region, 2,3,4,5,6-pentafluoroaniline shows two distinct peaks, at $2.86 \mu$ and $2.93 \mu$ (fig. 2-A). This is also true of 3-chloro-2,5,6trifluoro-4-( $\alpha, \alpha, \alpha$-trifluoromethyl)aniline, figure 2-C. In these aniline derivatives, the bands appear to be associated with the number of hydrogen atoms on the nitrogen atom. Replacing one of the hydrogen atoms by a methyl group merely eliminates one of the bands. This is also evident in figure 2-B, even though the compound contains two methylamino groups. Further evidence is obtained from the fact that 2,3,4,5,6-pentafluoro- $N, N$-dimethylaniline does not have an absorption band in this region. However, this near-infrared region does offer the possibility of distinguishing between primary, secondary, and tertiary fluoroanilines.

\section{Directional Effects}

A considerable number of disubstituted (nonfluorine groups) derivatives of tetrafluorobenzene have been prepared and their structures confirmed by unequivocal means. The directional effects determining the site of the second substitution in the electrophilic reaction of benzene has been the subject of much investigation. These effects are directly related to the charge distribution in the monosubstituted benzene and can be estimated from dipolemoment data [51]. Dipole moments of some fluorobenzenes have been reported [52]. The compounds studied do not, however, include any pentafluorobenzenes. The present work affords an insight into the character of the directional effects in polyfluorobenzenes. In table 1 are summarized these effects as observed from the reactions of various bases with a variety of monosubstituted pentafluorobenzenes. For example, the reaction of pentafluorobenzene, where $\mathrm{R}=\mathrm{H}$, with potassium hydroxide yields the 2,3,5,6-tetrafluorophenol. On further examination of table 1, it is apparent that, from the three possible isomers, the para product was obtained in most cases. This was true even though a great variety of conditions was used. It seems, therefore, that the isomer finally obtained depends on a number of involved parameters rather than on any single condition. From tabla 1, it is also evident that, in those cases where very reactive nucleophiles (such as the amide ion, lithium reagents, or alkoxides) were used, the para product was always isolated. These bases react to form salts with such functional groups as amines or hydroxyl groups, and no displacement of fluorine occurs.

With less reactive nucleophiles, such as aqueous hydroxides, ammonia, or alkylamines, isomers other than the para were also isolated. However, in these reactions, the isomer obtained probably depends to a large degree on the group (R) already present in the molecule, as well as on the nucleophile used. For example, the nitro group in pentafluoronitrobenzene exerts a powerful influence on the ortho positions, and, on reaction with ammonia, gives almost theoretical yields of 0 - and $p$-nitroamines in $7: 3$ ratio. Conversely, the amino group in pentafluoroaniline exhibits a strong influence in nucleophilic ractions at the meta position; as a result, the $m$-diamine is, with ammonia, the primary reaction-product. But the reaction of methylamine with pentafluoro- $N$-methylaniline yields only the para isomer, whereas the reaction of pentafluoro- $N, N$-dimethylaniline with dimethylamine yields the para, meta, and ortho isomers in a 10:4:1 ratio. Although the reactions of these amines appear to be "anomalous," a point could be made of the fact that the methyl- and dimethylamines are stronger nucleophiles than ammonia. In this case, then, the para structure would be the expected predominant isomer, which, indeed, it was. Similarly, it has been observed that the reaction of pentafluoro- $N, N$-dimethylaniline with ethoxide ion results in only the para product. It is likely that not only the attacking nucleophile but also the rasonance effects (due to nonfluorine groups) determine the isomer(s) that are obtained. The ease of fluorine displacement is probably increased by the ability of the solvent molecules to localize the electron density around this fluorine atom. Consideration must also be given to the possibility that resonance effects in the ground state may be altered in the transition state by the relative reactivity of the attacking nucleophile, thereby making possible the attack at a position other than the preferred site. For example, consider the following two structures

TABLE 1. Orientational effects of $\mathrm{C}_{6} \mathrm{~F}_{5} \mathrm{R}$ with nucleophiles a

\begin{tabular}{|c|c|c|c|c|c|c|c|c|c|c|c|c|}
\hline Base $R$ & $\mathrm{H}$ & $\mathrm{Br}$ & I & $\mathrm{CH}_{3}$ & $\mathrm{OCH}_{3}$ & ОФ & $\mathrm{OCH}_{2} \Phi$ & $\Phi$ & $\mathrm{NO}_{2}$ & $\mathrm{NH}_{2}$ & $\mathrm{NHCH}_{3}$ & $\mathrm{~N}\left(\mathrm{CH}_{3}\right)_{2}$ \\
\hline $\begin{array}{l}\mathrm{CH}_{3} \mathrm{ONa}_{2} \\
\mathrm{C}_{2} \mathrm{H}_{5} \mathrm{ONa} \\
\mathrm{C}_{6} \mathrm{H}_{5} \mathrm{ONa} \\
\mathrm{KOH} \\
\mathrm{NaSH} \\
\mathrm{CH}_{3} \mathrm{Li}_{2} \\
\mathrm{C}_{4} \mathrm{H}_{9} \mathrm{Li}_{2} \\
\mathrm{C}_{6} \mathrm{H}_{5} \mathrm{Li} \\
\mathrm{NaNH}_{2} \\
\mathrm{NH}_{3} \\
\mathrm{CH}_{3} \mathrm{NH}_{2} \\
\left(\mathrm{CH}_{3}{ }_{2} \mathrm{NH}_{2}\right. \\
\mathrm{NH}_{2} \mathrm{NH}_{2} \\
\mathrm{LiAlH}_{4}\end{array}$ & $\begin{array}{l}p \\
p \\
p\end{array}$ & $\begin{array}{c}p \\
p>0 \\
\mathrm{E}\end{array}$ & $p$ & $\begin{array}{l}p \\
p\end{array}$ & $\stackrel{p}{\mathrm{C}}$ & $p$ & $p$ & $p$ & $0>p$ & $m^{\mathrm{S}}>p$ & $p$ & $p>m>0$ \\
\hline
\end{tabular}

${ }^{a}{ }_{0}=\operatorname{orth} 0 ; m=$ meta $; p=$ para $; \mathrm{S}=$ salt formation; $\mathrm{C}=$ cleavage of ether moiety; $\mathrm{E}=\operatorname{exchange}\left(-78^{\circ} \mathrm{C}\right)$. 
from simple charge [2] effects:

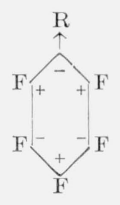

(1)

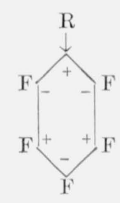

(2)
In (1), the incoming anion, $-\mathrm{R}_{1}$, would be expected to attack at the electron-deficient ortho and para positions, giving rise to either ortho or para products. In (1), if $\mathrm{R}=\mathrm{NO}_{2}$, the ground state could be visualized as shown, and the transition state as either (3) or (4).

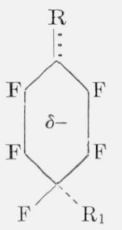

(3)

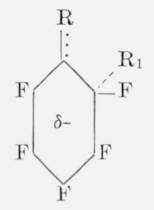

(4)
The $o$ - and $p$-nitroamines obtained indicate occurrence of this mode of reaction for pentafluoronitrobenzene, and it is doubtful whether use of a stronger nucleophile would alter the outcome. In (2), if $\mathrm{R}=\mathrm{NH}_{2}$ (an electron-donating group), the meta position relative to $\mathrm{R}$ appears to be the preferred site, and meta products would be expected. With a base such as ammonia, this is indeed the case. From consideration of the possible resonance structures (5) to (7a), it would appear that (7) is the predominant reacting species with a weak base, since the meta positions

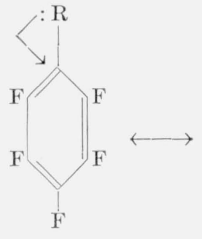

(5)

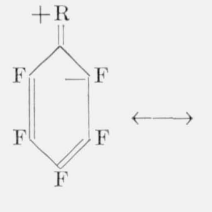

(6)

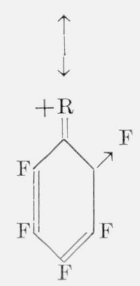

(6a)
(7)

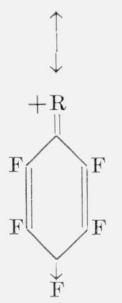

(7a)

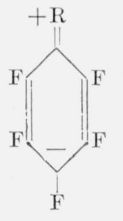

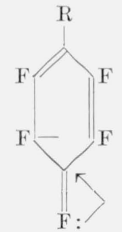

(8)

$\uparrow$

$\downarrow$

R

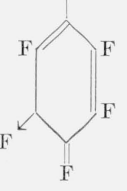

(8a) appear least negative. An alternative explanation could be offered in that a resonance structure, as in (8a), could be the reacting species. The induced charge would "prefer" to reside closer to the fluorine atom than to the carbon atom, and this effect would favor separation of a fluoride ion. Reaction would then be expected to occur at this site, giving rise to meta products. Effects similar to these have been used to explain the decrease in acidity of $p$-fluorophenol [53]. On the other hand, following similar reasoning, the resonance form (7a) would be expected to afford para products. This structure (7a) again appears to be the reacting species in the reaction of pentafluoro- $N$-methylaniline with methylamine, because only the para isomer was isolated. All three isomers were obtained with dimethylamine and pentafluoro- $N, N$-dimethylaniline. This indicates much less selectivity in structures (6a), (7a), and (8a), although the para isomer was obtained in greater proportions (somewhat surprisingly, since methylamine and dimethylamine have about equal basicity). In several other reactions also, all three isomers have been detected. Refluxing of pentafluorobenzene with lithium aluminum hydride in ether produces $o: m: p$ isomers of tetrafluorobenzene in 7:1:92 ratio, the orientational prefixes referring to the relative positions of the hydrogen atoms [54]. On treating pentafluorobenzene with ethanolic hydrazine, an $0: m: p$ distribution of $6: 1: 93$ is obtained [52]. Sodium hydrogen sulfide reacts with pentafluorobenzene in a glycol-pyridine mixture to yield only 2,3,5,6-tetrafluorothiophenol [55].

The reactions of pentafluoroanisole with bases yield additional information regarding directional effects of the anilines, since the amino and methoxyl groups have nearly equivalent influence on electrophilic substitution. However, the reaction of pentafluoroanisole with aqueous ammonia causes cleavage of the ether moiety. Resonance structures, such as (9), probably aid in the cleavage reaction.

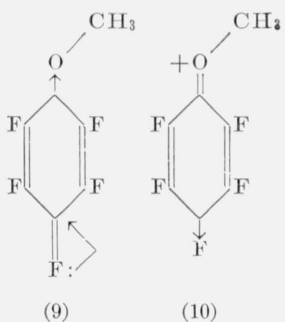

Other factors (such as solvent, and the ability of various groups to coordinate with the oxygen atom) also play an important role. The reaction with aqueous bases, therefore, simply becomes a competition between cleavage and replacement. With strong nucleophiles, such as amide ion or methoxide ion, only substitution occurs, presumably through a resonance form (10). In fact, such structures as (6a), (7a), and (10) appear to explain the majority of the products observed in nucleophilic reactions of aromatic fluorocarbons. For, if such forms as (8a), (6), and (7) were major contributing resonance forms, it should be possible to isolate meta products from most of the reactions encountered, which has not been the case. The effects, in general, do not show any specific differences qualitatively incompatible with the usual interpretations of directional effects in electrophilic ractions of monosubstituted benzenes. The predominance of para substitution 
suggests that the most important effect is simply an inductive one, since few of the monosubstituents can compete in electron withdrawability with fluorine. A much greater knowledge of the mechanisms of these reactions is needed before the directional effects can be satisfactorily classified.

\section{Experimental Procedure}

\subsection{Reaction With Hydroxides}

\section{a. 2,3,4,5,6-Pentafluorophenol}

In a $188-\mathrm{ml}$ bomb ware placed $40 \mathrm{~g}$ (0.207 mole) of hexafluorobenzene, $26.5 \mathrm{~g}$ (0.39 mole) of 85 percent potassium hydroxide, and $75 \mathrm{ml}$ of distilled water. The bomb was sealed and heated at $175{ }^{\circ} \mathrm{C}$ for $5 \mathrm{hr}$ with agitation. It was then cooled and opened, and the contents were filtered. The white salt of potassium pentafluorophenoxide hydrate was acidified with dilute hydrochloric acid, and the pentafluorophenol was separated from the aqueous phase. The aqueous solution was extracted several times with $50-\mathrm{ml}$ portions of dichloromethane. The organic layers were combined, dried (sodium sulfate), evaporated, and distilled. After removal of the solvent, there was obtained $33.1 \mathrm{~g}(83.5 \%)$ of pentafluorophenol, bp 144 to $145^{\circ} \mathrm{C}$.

\section{b. 2,3,5,6-Tetrafluorophenol}

In a 500-ml, three-necked flask were placed $67 \mathrm{~g}$ (0.4 mole) of pentafluorobenzene, $21.6 \mathrm{~g}$ (0.4 mole) of potassium hydroxide, $150 \mathrm{ml}$ of pyridine, and 2 $\mathrm{ml}$ of water. The contents were heated to reflux with vigorous stirring, and reflux was continued for $1 \mathrm{hr}$ after the hydroxide had dissolved. Then, 21.6 g $(0.4$ mole $)$ of potassium hydroxide was added through the condenser, and heating and stirring were continued for $24 \mathrm{hr}$. The pyridine was removed by distillation under reduced pressure, and the residue was recrystallized twice from water, yielding $70 \mathrm{~g}$ of potassium tetrafluorophenoxide dihydrate. The salt was acidified with dilute hydrochloric acid, the phenol was separated and dissolved in dichloromethane, and the solution was dried (sodium sulfate), evaporated, and distilled under reduced pressure, bp $47^{\circ} \mathrm{C} / 20 \mathrm{~mm}$. Yield: $21 \mathrm{~g}(32 \%$ based on pentafluorobenzene). The structure of the compound was verified by nuclear magnetic-resonance spectra due to fluorine atoms.

Analysis: Calculated for $\mathrm{C}_{6} \mathrm{H}_{2} \mathrm{~F}_{4} \mathrm{O}: \mathrm{C}, 43.3 ; \mathrm{H}$, 1.2. Found: C, 43.5; H, 1.5.

\section{c. 2,3,5,6-Tetrafluoro-p-cresol}

In a 500-ml, three-necked flask, a mixture of $36 \mathrm{~g}$ $(0.2 \mathrm{~mole})$ of pentafluorotoluene, $28 \mathrm{~g}(0.5 \mathrm{~mole})$ of potassium hydroxide, and $300 \mathrm{ml}$ of tert-butyl alcohol was refluxed, with stirring, for $2 \mathrm{hr}$. The flask was cooled and $100 \mathrm{ml}$ of water was added, after which $100 \mathrm{ml}$ of the alcohol was distilled off. This process was repeated until all of the alcohol had been removed, the temperature of the takeoff rising from 73 to $100{ }^{\circ} \mathrm{C}$. The distillation was continued until $6 \mathrm{~g}$ of pentafluorotoluene had been recovered. The residue was made acid with dilute hydrochloric acid, cooled, extracted with three $100-\mathrm{ml}$ portions of ether, and the extracts were dried (sodium sulfate) overnight. After evaporation of the ether, the black, oily residue was sublimed twice, yielding $12 \mathrm{~g}(32 \%)$ of white crystals; mp $52{ }^{\circ} \mathrm{C}$. Fluorine and proton absorption in the nuclear magnetic-resonance spectra confirmed the structure.

Analysis: Calculated for $\mathrm{C}_{7} \mathrm{~F}_{4} \mathrm{H}_{4} \mathrm{O}: \quad \mathrm{C}, 46.6 ; \mathrm{H}, 2.2$. Found: C, $46.3 ; \mathrm{H}, 2.4$.

\section{d. 2,3,5,6-Tetrafluoro-4-iodophenol}

To $20 \mathrm{~g}$ (0.068 mole) of pentafluoroiodobenzene in $100 \mathrm{ml}$ of pyridine were added $2 \mathrm{~g}(0.136 \mathrm{~mole})$ of potassium hydroxide and $1 \mathrm{ml}$ of water, and the mixture was refluxed for $4 \mathrm{hr}$. At the end of this period, an additional $2 \mathrm{~g}$ of potassium hydroxide was added and the mixture was refluxed for an additional $8 \mathrm{hr}$. After being cooled, $300 \mathrm{ml}$ of $10 \%$ aqueous hydrochloric acid was added, and the crude, yellow tetrafluoroiodophenol $(10.1 \mathrm{~g})$ was removed by filtration. Extraction of the aqueous layers with two 100-ml portions of dichloromethane gave an additional $2 \mathrm{~g}$ of the phenol. Net yield (based on pentafluoroiodobenzene, $1.5 \mathrm{~g}$ of which was recovered): 66 percent.

The tetrafluoroiodophenol was recrystallized from ether-petroleum ether as white plates, mp 79 to $81{ }^{\circ} \mathrm{C}$. Nuclear magnetic resonance showed this to be the para isomer; no other isomer was isolated. The near-infrared absorption spectrum showed only one hydroxyl peak, at $2.81 \mu$.

The benzoate was prepared in the usual way. Recrystallization from ether-petroleum ether, followed by vacuum sublimation $\left(60{ }^{\circ} \mathrm{C} / 1 \mathrm{~mm}\right)$, gave white microcrystals, mp 59 to $60.2^{\circ} \mathrm{C}$.

Analysis: Calculated for $\mathrm{C}_{13} \mathrm{H}_{5} \mathrm{~F}_{4} \mathrm{IO}_{2}$ : C, 39.41; H, 1.26; F, 19.1; I, 32.04. Found: C, 40.0; H, 1.35; F, 18.8; I, 31.9.

\section{e. Bromotetrafluorophenols}

The preparation of the bromotetrafluorophenols is essentially the same as that for the tetrafluoroiodophenol, except that the following quantities were used: $66 \mathrm{~g}$ (0.26 mole) of bromopentafluorobenzene, $150 \mathrm{ml}$ of pyridine, $1 \mathrm{ml}$ of water, and $28.5 \mathrm{~g}(0.53$ mole) of potassium hydroxide. After acidification of the reaction mixture, the products were extracted with three $100-\mathrm{ml}$ portions of dichloromethane. The combined extracts were dried (sodium sulfate) and the solvent was evaporated. Vacuum distillation of the residual liquid gave $10.5 \mathrm{~g}(16 \%)$ of unreacted bromopentafluorobenzene and two phenolic fractions.

Fraction 1: $31 \mathrm{~g}(56.3 \%)$; bp 73 to $75{ }^{\circ} \mathrm{C} / 2 \mathrm{~mm}$; mp 19 to $21{ }^{\circ} \mathrm{C}$; was shown by analytical, vaporphase chromatography (Viton A column at $175^{\circ} \mathrm{C}$ ) to be a mixture of two components in a $3: 1$ ratio.

One component was 2-bromo-3,4,5,6-tetrafluorophenol, $3.5 \mathrm{~g}(6.3 \%)$, mp 41 to $43{ }^{\circ} \mathrm{C}$. Nuclear magnetic-resonance spectra showed this compound to be the ortho isomer. This is also evident from 
the near-infrared spectra, which showed a split hydroxyl peak at $2.81 \mu$ and $2.84 \mu$, presumably due to the two types of hydrogen bonding that can occur.

The 3,5-dinitrobenzoate had $\mathrm{mp} 104$ to $105{ }^{\circ} \mathrm{C}$. Analysis: Calculated for $\mathrm{C}_{13} \mathrm{H}_{3} \mathrm{BrF}_{4} \mathrm{~N}_{2} \mathrm{O}_{6}$ : C, 35.6; H, 0.69; Br, 18.1. Found: C, 35.7; H, 0.7; Br, 18.7.

The second (and main) component of fraction 1 was 4-bromo-2,3,5,6-tetrafluorophenol, $11.5 \mathrm{~g}$ $(20.9 \%)$. The 3,5-dinitrobenzoate had mp 131 to $133{ }^{\circ} \mathrm{C}$.

Analysis: Calculated for $\mathrm{C}_{13} \mathrm{H}_{3} \mathrm{BrF}_{4} \mathrm{~N}_{2} \mathrm{O}_{6}$ : C, 35.6; H, 0.69; Br, 18.1. Found: C, 35.4; H, 0.6; Br, 20.3.

Fraction 2: $4 \mathrm{~g}(7.2 \%)$; bp 90 to $92^{\circ} \mathrm{C} / 2 \mathrm{~mm}$; $\mathrm{mp} 58$ to $60{ }^{\circ} \mathrm{C}$; was shown by nuclear magnetic resonance to be 4-bromo-2,3,5,6-tetrafluorophenol. Also, the near-infrared absorption spectrum showed only one peak in the $2.8-\mu$ region. The 3,5 -dinitrobenzoate had $\mathrm{mp} 133$ to $134{ }^{\circ} \mathrm{C}$. There was no depression of melting point on mixing the 3,5dinitrobenzoate of this fraction with that of the second component of fraction 1.

\section{f. 2-Chlorotrifluoro- $\alpha, \alpha, \alpha$-trifluoro-o-(and- $p$-)Cresols}

To $100 \mathrm{~g}$ (0.4 mole) of 2-chlorotetrafluoro- $\alpha, \alpha, \alpha$ trifluorotoluene in $100 \mathrm{ml}$ of pyridine were added $45.6 \mathrm{~g}$ (0.8 mole) of potassium hydroxide and $1 \mathrm{ml}$ of water. The mixture was slowly heated to 90 ${ }^{\circ} \mathrm{C}$ for $2 \mathrm{hr}$ with stirring, cooled, poured into 1 liter of 20 percent sulfuric acid, and allowed to settle overnight. It was filtered, and the bottom (fluorocarbon) layer was separated from the aqueous filtrate. The aqueous layer was further extracted with several $100-\mathrm{ml}$ portions of ether. The combined organic layers were dried (sodium sulfate) and the solvent removed, yielding $38 \mathrm{~g}(38.6 \%)$ of a mixture of two isomeric cresols, bp 97 to $103{ }^{\circ} \mathrm{C} / 15$ $\mathrm{mm}$. The components were separated by preparative vapor-phase chromatography using a Viton A column at $190{ }^{\circ} \mathrm{C}$. The first compound eluted was 2-chlorotrifluoro- $\alpha, \alpha, \alpha$-trifluoro-o-cresol: $0.5 \mathrm{~g}$; bp 92 to $93{ }^{\circ} \mathrm{C} / 15 \mathrm{~mm} ; \mathrm{n}_{\mathrm{D}}^{24} 1.4510$; infrared absorption showed a split hydroxyl band, at $2.76 \mu$ and $2.81 \mu$. The second compound was 2-chlorotrifluoro- $\alpha, \alpha, \alpha$ trifluoro-p-cresol: $15 \mathrm{~g}$; bp 102 to $103{ }^{\circ} \mathrm{C} / 15 \mathrm{~mm}$; $\mathrm{n}_{\mathrm{D}}^{24} 1.4510$; infrared absorption showed only one (strong) hydroxyl band, at $2.81 \mu$.

\subsection{Reactions With Alkoxides}

\section{a. 4-Bromo-2,3,5,6-tetrafluoroanisole}

A solution of $11.5 \mathrm{~g}(0.5 \mathrm{~g}$-atom $)$ of sodium in 150 $\mathrm{ml}$ of anhydrous methanol was added dropwise to a stirred solution of $123.5 \mathrm{~g}(0.5$ mole $)$ of bromopentafluorobenzene in $70 \mathrm{ml}$ of dry pyridine at reflux temperature. The addition required $1.5 \mathrm{hr}$; refluxing was continued for an additional $15 \mathrm{hr}$. The mixture was cooled and poured into 1 liter of 10 percent aqueous hydrochloric acid. The usual isolation, as described above, gave $89 \mathrm{~g}(68.8 \%)$ of bromotetrafluoroanisole, bp 76 to $79{ }^{\circ} \mathrm{C} / 5 \mathrm{~mm}$. Analytical vapor-phase chromatography (Viton A column at $175^{\circ} \mathrm{C}$ ) showed slight contamination of the product with another compound. Separation was accomplished by preparative scale vapor-phase chromatography, using 10-g samples (Viton A column at $175^{\circ} \mathrm{C}$ ). 'The fore-cut, $8 \mathrm{~g}$, still gave two peaks. The (main) second fraction, $66 \mathrm{~g}$, showed only one peak in analytical vapor-phase chromatography; bp 79 to $81^{\circ} \mathrm{C} / 5 \mathrm{~mm}$; $\mathrm{n}_{\mathrm{D}}^{25} 1.4812$. Nuclear magnetic resonance showed this to be the para isomer.

Analysis: Calculated for $\mathrm{C}_{7} \mathrm{H}_{3} \mathrm{BrF}_{4} \mathrm{O}$ : C, $32.4 ; \mathrm{H}$, $1.2 ; \mathrm{Br}, 30.9$. Found: C, $32.7 ; \mathrm{H}, 1.3 ; \mathrm{Br}, 31.9$.

A higher-boiling fraction $(2 \mathrm{~g}), 72$ to $76{ }^{\circ} \mathrm{C} / 2 \mathrm{~mm}$, had three components; these were not investigated further.

\section{b. 2,3,5,6-Tetrafluoro-4-iodoanisole}

To a stirred solution of $10 \mathrm{~g}(0.034$ mole $)$ of pentafluoroiodobenzene and $50 \mathrm{ml}$ of dry pyridine, heated to reflux temperature, was added a solution of $0.8 \mathrm{~g}$ (0.034 g-atom) of sodium in $15 \mathrm{ml}$ of anhydrous methanol. After being refluxed for $3 \mathrm{hr}$, the cooled solution was poured into $100 \mathrm{ml}$ of $6 \mathrm{~N}$ aqueous hydrochloric acid. The orange (fluorocarbon) layer was separated, and the aqueous layer extracted twice with $50-\mathrm{ml}$ portions of ether. The combined organic layers were dried (sodium sulfate), evaporated, and distilled. After removal of the unreacted pentafluoroiodobenzene $(1.5 \mathrm{~g})$, there was obtained $5.5 \mathrm{~g}(63 \%$ based on reacted pentafluoroiodobenzene) of 2,3,5,6-tetrafluoro-4-iodoanisole; bp 113 to $115^{\circ} \mathrm{C} / 20 \mathrm{~mm} ; \mathrm{n}_{\mathrm{D}}^{22} 1.5229$; the structure was confirmed by nuclear magnetic resonance. Analytical, vapor-phase chromatography (at $190{ }^{\circ} \mathrm{C}$ ) showed a 3-percent contamination with starting material. The sample was redistilled before elementary analysis.

Analysis: Calculated for $\mathrm{C}_{7} \mathrm{H}_{3} \mathrm{~F}_{4} \mathrm{IO}$ : C, 27.48; H, 0.98 ; F, 24.83; I, 41.48. Found: C, 27.8; H, 1.0; F, $24.4 ;$ I, 41.0 .

\section{c. Octafluoro-4,4'-dimethoxybiphenyl}

A mixture of $1 \mathrm{~g}$ of 2,3,5,6-tetrafluoro-4-iodoanisole and $1 \mathrm{~g}$ of activated copper powder was gently refluxed for $12 \mathrm{~min}$, cooled, and extracted first with $10 \mathrm{ml}$ of acetone and then with $10 \mathrm{ml}$ of benzene. The combined extracts were filtered and the solvents evaporated. The yellow solid obtained was decolorized with charcoal, and recrystallized from petroleum ether. There was obtained $0.2 \mathrm{~g}(17 \%)$ of octafluoro- $4,4^{\prime}$-dimethoxybiphenyl as white plates; mp 90 to $91.2{ }^{\circ} \mathrm{C}$.

Analysis: Calculated for $\mathrm{C}_{14} \mathrm{H}_{5} \mathrm{~F}_{8} \mathrm{O}_{2}$ : C, 46.94; H, 1.69 ; F, 42.44. Found: C, 47.5; H, 1.88; F, 42.8.

\section{d. Benzyl Pentafluorophenyl Ether}

Method A. In a 500-ml flask equipped with stirrer and reflux condenser, $9 \mathrm{~g}$ (0.4 g-atom) of sodium was treated with $250 \mathrm{ml}$ of benzyl alcohol, and the solution cooled overnight. Then, $75 \mathrm{~g}$ (0.41 mole) of hexafluorobenzene was added, stirring was resumed, and the mixture slowly heated to the reflux temperature of hexafluorobenzene. Heating and stirring were continued for $24 \mathrm{hr}$, and then the mixture was cooled overnight with stirring. The 
precipitate was filtered off with suction, the filtrate was concentrated, ethanol was added, and the resulting solution was refrigerated. The resulting precipitate was recrystallized until a constant melting point $\left(44{ }^{\circ} \mathrm{C}\right)$ was obtained; yield, $30 \mathrm{~g}$ $(28 \%)$ of white crystals.

Method B. In a 500-ml flask, $4.6 \mathrm{~g}$ (0.2 mole) of sodium was added to a solution of $22 \mathrm{~g}$ of benzyl alcohol in $250 \mathrm{ml}$ of tert-butyl alcohol. The solution was cooled overnight and $40 \mathrm{~g}$ of hexafluorobenzene (0.22 mole) was added. The solution was stirred and refluxed for $40 \mathrm{hr}$, after which the alcohols were distilled off. The product was recrystallized twice from ethanol; vield, $33 \mathrm{~g}(60 \%): \mathrm{mp} 44{ }^{\circ} \mathrm{C}$.

Analysis: Calculated for $\mathrm{C}_{13} \mathrm{~F}_{5} \mathrm{H}_{7} \mathrm{O}: \mathrm{C}, 56.9 ; \mathrm{H}$, 2.6. Found: C, 56.8; H, 2.6. e. 2,3,4,5,6-Pentafluorophenyl Ether and 2,3,5,6-Tetrafluoro-
1,4-diphenoxybenzene

Method A. To $8 \mathrm{~g}$ (0.043 mole) of hexafluorobenzene in $30 \mathrm{ml}$ of $N, N$-dimethylformamide was added $5.28 \mathrm{~g}$ (0.04 mole) of potassium phenoxide in one portion at room temperature. After the initial reaction had ceased, the mixture was heated at $120{ }^{\circ} \mathrm{C}$ for $0.5 \mathrm{hr}$, cooled, and diluted with $100 \mathrm{ml}$ of water. The bottom (fluorocarbon) layer eventually solidified, and the white solid was separated by filtration. After drying, and fractional sublimation at $70{ }^{\circ} \mathrm{C} / 1 \mathrm{~mm}$, there was obtained $3.5 \mathrm{~g}(31.3 \%)$ of 2,3,4,5,6-pentafluorophenyl phenyl ether, $\mathrm{mp} 28$ to $29{ }^{\circ} \mathrm{C}$. This compound was identical with that described under Method B.

There was also obtained $1 \mathrm{~g}(7 \%)$ of $2,3,5,6-$ tetrafluoro-1,4-diphenoxybenzene as white crystals, mp 147 to $149{ }^{\circ} \mathrm{C}$.

Analysis: Calculated for $\mathrm{C}_{18} \mathrm{H}_{10} \mathrm{~F}_{4} \mathrm{O}_{2}: \mathrm{C}, 64.6 ; \mathrm{H}$, 2.99; F, 22.7. Found: C, 64.75; H, 2.98; F, 23.1.

Method B. In a $43-\mathrm{ml}$ bomb were placed $11 \mathrm{~g}$ (0.05 mole) of potassium pentafluorophenoxide, $15 \mathrm{~g}$ (0.10 mole) of bromobenzene, and $1 \mathrm{~g}$ of copper which had been activated by Vogel's method [56]. The bomb was heated to $210^{\circ} \mathrm{C}$ with rocking, maintained at this temperature for $6 \mathrm{hr}$, and rocked overnight as it cooled to room temperature. The mixture was filtered and the filtrate distilled through a short column. After the excess bromobenzene had been removed, $2 \mathrm{~g}(15.5 \%$, based on the potassium salt of $2,3,4,5,6$-pentafluorophenol) of $2,3,4,5,6$ pentafluorophenyl phenyl ether was obtained, which boiled at $240{ }^{\circ} \mathrm{C}$ and solidified slightly below room temperature.

Analysis: Calculated for $\mathrm{C}_{12} \mathrm{H}_{5} \mathrm{~F}_{5} \mathrm{O}: \mathrm{C}, 55.4 ; \mathrm{H}$, 1.9. Found: C, 55.7; H, 2.1.

\section{f. Perfluorophenyl Ethers}

To $30 \mathrm{ml}$ of $N, N$-dimethylformamide was added $6 \mathrm{~g}(0.027 \mathrm{~mole})$ of potassium pentafluorophenoxide and $12.8 \mathrm{~g}$ (0.069 mole) of hexafluorobenzene; heat was not evolved. After being refluxed for $14 \mathrm{hr}$, the black solution was poured into $100 \mathrm{ml}$ of water, and the mixture was extracted with several 50 -ml portions of ether. The organic layer was separated, dried (sodium sulfate), and evaporated. After the removal of $4.5 \mathrm{~g}$ of unreacted hexafluorobenzene, the residual solid was sublimed at $50{ }^{\circ} \mathrm{C} / 50 \mathrm{~mm}$ to give $1.5 \mathrm{~g}$ $(16 \%)$ of perfluorophenyl ether; white crystals, mp 67 to $69^{\circ} \mathrm{C}$. Confirmation of the structure was obtained from mass-spectrometer analysis, which showed the parent peak at 350 mass units.

Analysis: Calculated for $\mathrm{C}_{12} \mathrm{~F}_{10} \mathrm{O}: \mathrm{C}, 41.15 ; \mathrm{F}$, 54.3. Found: C, 41.53; F, 53.0; H, 0.0.

A second product was obtained from the sublimation; $\mathrm{mp} 145$ to $148{ }^{\circ} \mathrm{C}$. Although its structure has not been confirmed, it may be $p$-bis(pentafluorophenoxy)2,3,5,6-tetrafluorobenzene.

\section{g. 4-Ethoxy-2,3,5,6-tetrafluoro- $N, N$-dimethylamine}

In a $100-\mathrm{ml}$ flask, $0.6 \mathrm{~g}$ of sodium was dissolved in $50 \mathrm{ml}$ of absolute ethanol and the solution was refluxed for $1 \mathrm{hr}$. To this solution was added $5.3 \mathrm{~g}$ (0.025 mole) of 2,3,4,5,6-pentafluoro- $N, N$-dimethylaniline, and the mixture was refluxed for $2 \mathrm{hr}$ and poured into an excess of water. The organic layer was separated, washed with water, dried, and distilled. Yield: $5.1 \mathrm{~g}(85 \%)$ of a clear, colorless liquid boiling at $34^{\circ} \mathrm{C} / 1 \mathrm{~mm}$.

Analysis: Calculated for $\mathrm{C}_{10} \mathrm{H}_{11} \mathrm{~F}_{4} \mathrm{NO}: \mathrm{C}, 50.6 ; \mathrm{H}$, $4.7 ; \mathrm{N}, 5.9 ; \mathrm{F}, 32.1$. Found: C, 50.8; H, 5.0; N, 5.9 .

\subsection{Reactions With Amines}

\section{a. 2,3,4,5,6-Pentafluoroaniline}

The reactions using ammonia or amines were carried out in a stainless-steel, silver-lined bomb. A mixture of $280 \mathrm{~g}(1.5 \mathrm{~mole})$ of hexafluorobenzene and $400 \mathrm{ml}$ of 28 -percent aqueous ammonia was sealed in an $800-\mathrm{ml}$ bomb, which was placed in a heater-rocker mechanism previously heated to $235^{\circ} \mathrm{C}$. The bomb was rocked for $2 \mathrm{hr}$ at $235^{\circ} \mathrm{C}$, removed, and rapidly cooled to room temperature in running water. The contents were poured into a large beaker, and the aqueous layer was separated and extracted three times with ether. The ether extracts were dried (sodium sulfate), and evaporated. The substituted aniline obtained in this way was added to the oily layer from the bomb, and the mixture was evacuated in a vacuum desiccator for $8 \mathrm{hr}$, the volatile materials, mostly hexafluorobenzene and ammonia, being collected in a dry ice - acetone trap. The residual mass of brown crystals was partially sublimed at atmospheric pressure onto a cold-finger condenser, to yield $236 \mathrm{~g}(86 \%)$ of white crystals of pentafluoroaniline; $\mathrm{mp} 34^{\circ} \mathrm{C}$.

The remainder of residue was heated to about 75 ${ }^{\circ} \mathrm{C}$ at less than $1 \mathrm{~mm}$ pressure. White crystals lacking a definite melting point sublimed onto the cold finger. Yield: $28 \mathrm{~g}(10 \%)$. Elementary analysis indicated it to be tetrafluorophenylenediamine, and nuclear magnetic resonance indicated it to be the meta isomer contaminated with a small proportion of the para isomer.

Analysis: Calculated for $\mathrm{C}_{6} \mathrm{H}_{4} \mathrm{~F}_{4} \mathrm{~N}_{2}$ : C, 40.0; $\mathrm{H}$, $2.2 ; \mathrm{N}, 15.5$. Found: C, 40.2; H, 2.4; N, 15.2 . 


\section{b. 2,3,4,5,6-Pentafluoro- $N$-methylaniline}

In a 200-ml bomb, $56 \mathrm{~g}$ of hexafluorobenzene and $110 \mathrm{ml}$ of 30-percent aqueous methylamine were heated at $220{ }^{\circ} \mathrm{C}$ for $3 \mathrm{hr}$ with continuous rocking. The products were dissolved in ether and the ether was evaported. The pentafluoro- $N$-methylaniline was distilled at atmospheric pressure, and redistilled under reduced pressure. Yield; 59 percent; bp 170 to $172{ }^{\circ} \mathrm{C}$ at $760 \mathrm{~mm}$.

Analysis: Calculated for $\mathrm{C}_{7} \mathrm{H}_{4} \mathrm{~F}_{5} \mathrm{~N}: \mathrm{C}, 42.6 ; \mathrm{H}$, $2.1 ; \mathrm{N}, 7.1$. Found: C, 42.7; H, 2.2; N, 7.0.

The residue was crystallized from absolute alcohol, yielding $2,3,5,6$-tetrafluoro- $N, N^{\prime}$-dimethylphenylenediamine, which was purified by sublimation and recrystallization. Yield: 25 percent; $\mathrm{mp} 94{ }^{\circ} \mathrm{C}$.

Analysis: Calculated for $\mathrm{C}_{8} \mathrm{H}_{8} \mathrm{~F}_{4} \mathrm{~N}_{2}$ : C, 46.2; H, 3.8 ; N, 13.5. Found: C, 46.1; H, 3.9; N, 13.6.

\section{c. 2,3,4,5,6-Pentafluoro- $N, N$-dimethylaniline}

A mixture of $110 \mathrm{ml}$ of 25-percent aqueous dimethylamine and $50 \mathrm{~g}$ of hexafluorobenzene was heated at $235{ }^{\circ} \mathrm{C}$ for $2 \mathrm{hr}$. The reaction products were purified as described for pentafluoroaniline. A colorless liquid which distilled at $88{ }^{\circ} \mathrm{C} / 1 \mathrm{~mm}$ was pentafluoro- $N, N$-dimethylaniline $(65 \%$ of the oily layer). Four additional fractions were collected.

Analysis: Calculated for $\mathrm{C}_{8} \mathrm{H}_{6} \mathrm{~F}_{5} \mathrm{~N}$ : C, 45.6; H, $2.6 ; \mathrm{N}, 6.6$. Found: C, 45.7; H, 2.8; N, 6.5.

Fraction 2, bp 88 to $126{ }^{\circ} \mathrm{C} / 1 \mathrm{~mm}$, was identified by vapor-phase chromatography to be a mixture of dimethylaniline with three other compounds. Fraction 3, bp 126 to $134{ }^{\circ} \mathrm{C} / 1 \mathrm{~mm}$, contained the three isomers of bis(dimethylamino)tetrafluorobenzene, with the meta isomer predominant. Fraction 4, bp 134 to $140{ }^{\circ} \mathrm{C} / 1 \mathrm{~mm}$, contained equal amounts of $m$ and $p$-isomer. Fraction 5, bp 140 to $148^{\circ} \mathrm{C} / 1 \mathrm{~mm}$, was almost pure $p$-isomer. Analysis for $\mathrm{C}, \mathrm{H}$, and $\mathrm{N}$ indicated the same elementary composition for all fractions.

Analysis: Calculated for $\mathrm{C}_{10} \mathrm{H}_{12} \mathrm{~F}_{4} \mathrm{~N}_{2}$ : C, 50.9; H, $5.1 ; \mathrm{N}, 11.8$. Found: C, 50.9; H, 5.1; N. 11.7 .

Nuclear magnetic resonance analysis showed that the predominant isomer in Fraction 3 had three different fluorine-bond peaks, one twice as large as the other two, indicating the meta isomer; the highboiling isomer (Fraction 5) showed only one fluorine bond and was, therefore, the para compound. The meta and para isomers were obtained relatively pure by vapor-phase chromatography; the ortho isomer was present in such small proportion as to warrant no further purification.

\section{d. $p$-Bromotetrafluoroaniline}

Thirty grams of bromopentafluorobenzene and 70 $\mathrm{ml}$ of 28-percent ammonium hydroxide were placed in a $110-\mathrm{ml}$ bomb, heated to $200{ }^{\circ} \mathrm{C}$, and shaken at this temperature for $2 \mathrm{hr}$. The product was purified as previously described for pentafluoroaniline. The yield of crystalline product, $\mathrm{mp} 61{ }^{\circ} \mathrm{C}$, was $22 \mathrm{~g}$ $(77 \%)$. Nuclear magnetic resonance indicated that the compound was the para isomer.
Analysis: Calculated for $\mathrm{C}_{6} \mathrm{H}_{2} \mathrm{BrF}_{4} \mathrm{~N}$ : C, 29.5; H, $0.8 ; \mathrm{N}, 5.7 ; \mathrm{Br}, 32.7$. Found: C, 29.4; H, 0.8; N, $5.8 ; \mathrm{Br}, 32.8$.

\section{e. Tetrafluoro-p-iodoaniline}

Sixteen grams of pentafluoroiodobenzene and $30 \mathrm{ml}$ of 8-percent aqueous ammonium hydroxide were heated to $165^{\circ} \mathrm{C}$ in a $43-\mathrm{ml}$ bomb and shaken at this temperature for $2 \mathrm{hr}$. The product was purified as described previously, except that, after sublimation, the compound was recrystallized from petroleum ether, yielding $7.6 \mathrm{~g}(46.7 \%)$ of slightly yellow crystals; $\mathrm{mp} 77^{\circ} \mathrm{C}$. Nuclear magnetic-resonance spectra indicated it to be the para isomer.

Analysis: Calculated for $\mathrm{C}_{6} \mathrm{H}_{2} \mathrm{~F}_{4} \mathrm{IN}$ : C, $24.7 ; \mathrm{H}$, $0.7 ; \mathrm{N}, 4.8 ; \mathrm{I}, 43.6$. Found: C, $24.7 ; \mathrm{H}, 0.8 ; \mathrm{N}, 4.6$; I, 42.9 .

\section{f. $p$-(Benzyloxy)tetrafluoroaniline}

Twenty grams of benzyl pentafluorophenyl ether and a large excess of 28 -percent aqueous ammonium hydroxide were heated in a $110-\mathrm{ml}$ bomb to $160{ }^{\circ} \mathrm{C}$ and shaken at this temperature for $2 \mathrm{hr}$. A small amount of white crystals was observed clinging to the inside of the bomb. This material was removed and recrystallized three times from ethanol. Yield: $3 \mathrm{~g}(13 \%)$ of slightly yellow needles, mp $97{ }^{\circ} \mathrm{C}$; it was shown by nuclear magnetic resonance to be the para isomer.

Analysis: Calculated for $\mathrm{C}_{13} \mathrm{H}_{9} \mathrm{~F}_{4} \mathrm{NO}: \mathrm{C}, 53.1 ; \mathrm{H}$, $3.3 ; \mathrm{N}, 5.1$. Found: C, $53.0 ; \mathrm{H}, 3.4 ; \mathrm{N}, 4.9$.

\section{g. 2-Chlorotrifluoro- $\alpha, \alpha$, $\alpha$-trifluoro-p-toluidine}

A mixture of $50 \mathrm{~g}$ of 2-chlorotetrafluoro- $\alpha, \alpha, \alpha$ trifluoro-toluene and $120 \mathrm{ml}$ of 28 -percent ammonium hydroxide was heated to $210{ }^{\circ} \mathrm{C}$ in a $200-\mathrm{ml}$ bomb and shaken at this temperature for $2 \mathrm{hr}$; the crude substituted toluidine was purified by sublimation, giving $22 \mathrm{~g}(43 \%)$ of white crystals which readily decomposed at room temperature in the presence of air (accounting for discrepancies in the analysis).

Analysis: Calculated for $\mathrm{C}_{7} \mathrm{H}_{2} \mathrm{ClF}_{6} \mathrm{~N}: \mathrm{C}, 33.7 ; \mathrm{H}$, $0.8 ; \mathrm{N}, 5.6 ; \mathrm{Cl}, 14.2$. Found: $\mathrm{C}, 32.6 ; \mathrm{H}, 1.1 ; \mathrm{N}, 6.2$; $\mathrm{Cl}, 14.9$.

\section{h. 2,3,5,6-Tetrafluoro-p-anisidine}

To $100 \mathrm{ml}$ of anhydrous liquid ammonia and $0.1 \mathrm{~g}$ of ferric nitrate at $-70{ }^{\circ} \mathrm{C}$ was added, in small pieces, $2.99 \mathrm{~g}$ (0.13 g-atom) of sodium. When the blue color had permanently disappeared, $25 \mathrm{~g}$ ( 0.13 mole) of pentafluoroanisole was added slowly during $45 \mathrm{~min}$. The mixture was stirred for $5 \mathrm{hr}$ at $-70{ }^{\circ} \mathrm{C}$, and the ammonia was allowed to evaporate overnight at room temperature. After the addition of $100 \mathrm{ml}$ of water, the fluorocarbon layer was separated, and the aqueous layer was extracted with three $50-\mathrm{ml}$ portions of dichloromethane. The unreacted pentafluoroanisole, $7 \mathrm{~g}(28 \%)$, was removed by distillation; bp 137 to $138{ }^{\circ} \mathrm{C}$. By vacuum sublimation $\left(80{ }^{\circ} \mathrm{C} / 1 \mathrm{~mm}\right)$ of the residue, tetrafluoro- $p$ anisidine, $2.8 \mathrm{~g}$ (14\% based on reacted penta- 
fluoroanisole), was obtained as white needles, mp 75 to $76.5{ }^{\circ} \mathrm{C}$; the structure was identified by nuclear magnetic resonance.

Analysis: Calculated for $\mathrm{C}_{7} \mathrm{H}_{5} \mathrm{~F}_{4} \mathrm{NO}$ : C, 43.05; $\mathrm{H}, 2.56 ; \mathrm{N}, 7.19$. Found: C, 43.10; H, 2.7; N, 7.1. After removal of the above compound, the residue liquefied, and $1.2 \mathrm{~g}(6.7 \%)$ of solid 4,4'-dimethoxyoctafluorodiphenylamine was obtained by distillation, bp 80 to $82{ }^{\circ} \mathrm{C} / 1 \mathrm{~mm}$. Sublimation at $80{ }^{\circ} \mathrm{C} / 1$ $\mathrm{mm}$, and recrystallization from petroleum ether gave white needles, mp 78 to $79{ }^{\circ} \mathrm{C}$. The melting point of a mixture of the fluoroanisidine and the diphenylamine compound was depressed. Nuclear magnetic resonance showed that a substituted anisyl group was linked to the nitrogen atom at the para position.

Analysis: Calculated for $\mathrm{C}_{14} \mathrm{H}_{7} \mathrm{~F}_{8} \mathrm{NO}_{2}$ : C, 45.0; H, 1.88; N, 3.75. Found: C, 45.4; H, 2.0; N, 3.5.

The residue gave $4,4^{\prime}, 4^{\prime \prime}$-trimethoxydodecafluorotriphenylamine as a yellow oil; $2.2 \mathrm{~g}$; bp 157 to $159{ }^{\circ} \mathrm{C} / 1 \mathrm{~mm} ; \mathrm{n}_{\mathrm{D}}^{23} 1.5005$.

Analysis: Calculated for $\mathrm{C}_{21} \mathrm{H}_{9} \mathrm{~F}_{12} \mathrm{NO}_{3}$ : C, 46.4; $\mathrm{H}, 1.66 ; \mathrm{N}, 2.57$. Found $\mathrm{C}, 46.7 ; \mathrm{H}, 2.1 ; \mathrm{N}, 2.50$.

\subsection{Diazotization of Pentafluoroaniline}

\section{a. Pentafluoroiodobenzene}

In a 250-ml, 3-necked, Monel flask (fitted with a metal inlet tube, Monel reflux condenser, and Tefloncovered, magnetic stirring-bar) was placed $20 \mathrm{~g}$ (0.108 mole) of pentafluoroaniline. Approximately $75 \mathrm{ml}$ of anhydrous hydrogen fluoride was condensed into the flask at $-20^{\circ} \mathrm{C}$ and $7.27 \mathrm{~g}$ ( 0.105 mole $)$ of sodium nitrite was added during $30 \mathrm{~min}$. The flask was allowed to warm to $-10{ }^{\circ} \mathrm{C}$, stirring was continued for an additional $1 \mathrm{hr}, 17.6 \mathrm{~g}$ (0.106 mole) of granular potassium iodide was added during $30 \mathrm{~min}$, and the mixture was allowed to warm to $25^{\circ} \mathrm{C}$ in $1 \mathrm{hr}$. The residual hydrogen fluoride was removed by warming the flask at $50{ }^{\circ} \mathrm{C}$ and the mixture was poured into $100 \mathrm{ml}$ of water in a Monel beaker. The organic layer was separated, washed with three $50-\mathrm{ml}$ portions of saturated sodium bicarbonate solution and water, and dried (sodium sulfate). On distillation, $16.5 \mathrm{~g}(50 \%)$ of pentafluoroiodobenzene, bp 77 to $79{ }^{\circ} \mathrm{C} / 35 \mathrm{~mm}$, was obtained. Analytical, vapor-phase chromatography (Viton A column at $150^{\circ} \mathrm{C}$ ) showed only one product, with a retention time identical with that of pentafluoroiodobenzene.

Bromopentafluorobenzene $(35 \%$ yield) was prepared by the same procedure, except that $12 \mathrm{~g}$ of potassium bromide and $15 \mathrm{~g}$ of cuprous bromide was used in place of the potassium iodide.

\subsection{Oxidation of Pentafluoroaniline}

\section{a. Decafluoroazoxybenzene}

In a $250-\mathrm{ml}$ flask were placed $10 \mathrm{~g}$ (0.055 mole) of pentafluoroaniline, $100 \mathrm{ml}$ of glacial acetic acid, and $25 \mathrm{ml}$ (0.22 mole) of 30-percent hydrogen peroxide. At $25{ }^{\circ} \mathrm{C}$, the solution turned blue, green, dark brown, yellow, red, and finally orange within $24 \mathrm{hr}$. During an additional $24 \mathrm{hr}$, an orange liquid separated on the bottom. The reaction was stopped by dilution with $300 \mathrm{ml}$ of water. The orange liquid was separated and, when chilled in ice, solidified. Yield: $4.5 \mathrm{~g}(22 \%)$. On sublimation at $80^{\circ} \mathrm{C} / 1 \mathrm{~mm}$, followed by recrystallization (decolorization with charcoal) from petroleum ether, white plates of decafluoroazoxybenzene, $\mathrm{mp} 53$ to $54{ }^{\circ} \mathrm{C}$, formed. On standing, the material acquired a green tint. Confirmation of the structure was obtained from mass-spectrometer analysis, which showed the parent mass peak at 378. The ultraviolet absorption spectrum showed two maxima, one at $230 \mathrm{~m} \mu$ and the other at $296 \mathrm{~m} \mu$.

\section{b. Decafluoroazobenzene}

A mixture of $5 \mathrm{~g}$ (0.013 mole) of decafluoroazoxybenzene, $15 \mathrm{~g}$ of zinc powder, $5 \mathrm{~g}$ of ammonium chloride, $10 \mathrm{ml}$ of water, and $75 \mathrm{ml}$ of 95 -percent ethanol was stirred under reflux for $30 \mathrm{~min}$. The mixture was filtered hot, and the zinc was extracted with two $50-\mathrm{ml}$ portions of hot alcohol. The alcoholic filtrates were poured onto $100 \mathrm{~g}$ of ice, and the tan precipitate was filtered off and dried. Sublimation at $60{ }^{\circ} \mathrm{C} / 1 \mathrm{~mm}$ gave $2 \mathrm{~g}(41.5 \%)$ of orange decafluoroazobenzene, $\mathrm{mp} 57$ to $59{ }^{\circ} \mathrm{C}$. Confirmation of the structure was obtained from mass-spectrometer analysis, which showed the parent mass peak at 362 . The ultraviolet absorption spectrum showed one peak at $230 \mathrm{~m} \mu$, but this had two slight inflections, one at $280 \mathrm{~m} \mu$ and the other $326 \mathrm{~m} \mu$. Solutions of decafluoroazoxybenzene are yellow and show one absorption peak at $370 \mathrm{~m} \mu$ in the visible range, whereas the solutions of decafluoroazobenzene are orange and absorb at $465 \mathrm{~m} \mu$.

\subsection{Reactions With Organolithium Compounds}

\section{a. 2,3,4,5,6-Pentafluorotoluene}

Methyllithium was prepared in a $500-\mathrm{ml}$, threenecked flask equipped with a dropping funnel, magnetic stirrer, and a fitting for introducing a continuous flow of argon. Clean lithium, $4.5 \mathrm{~g}$ ( 0.61 mole) cut into small pieces, and $200 \mathrm{ml}$ of sodium-dried ether were placed in the flask. A solution of $43 \mathrm{~g}$ of iodomethane in $50 \mathrm{ml}$ of absolute ether was added dropwise to the mixture while vigorous stirring and a constant atmosphere of argon were maintained. After the reaction of the iodomethane with the lithium had begun (as evidenced by a clouding of the ether), the mixture was cooled to $-10^{\circ} \mathrm{C}$ with dry ice-acetone. The temperature was maintained between $-10{ }^{\circ} \mathrm{C}$ and $-20{ }^{\circ} \mathrm{C}$ during the entire preparation of the methyllithium.

The ether solution of methyllithium was transferred to a dropping funnel while under an argon atmosphere, and was added dropwise to a solution of $60 \mathrm{~g}$ of hexafluorobenzene in $250 \mathrm{ml}$ of dry pentane initially at room temperature, slight refluxing from the heat of reaction being maintained. Continuous stirring under an argon atmosphere was maintained throughout the addition and for an additional $17 \mathrm{hr}$; during the final $2 \mathrm{hr}$, the solution was refluxed gently. After being cooled to room temperature, the suspension was filtered and the filtrate was washed with water and dried (sodium sulfate). The liquid was 
distilled through a packed column. Yield: $34 \mathrm{~g}$ $(70 \%)$, bp $115^{\circ} \mathrm{C}$.

Analysis: Calculated for $\mathrm{C}_{7} \mathrm{H}_{3} \mathrm{~F}_{5}: \mathrm{C}, 46.2 ; \mathrm{H}, 1.6$. Found: C, 46.4; H, 1.8.

\section{b. Butyl-2,3,4,5,6-pentafluorobenzene}

In a $250-\mathrm{ml}$, three-necked flask (fitted with a dropping funnel, stirrer, and reflux condenser, and containing a helium atmosphere) were placed $1.86 \mathrm{~g}$ $(0.268 \mathrm{~g}$-atom $)$ of $1 / 8-\mathrm{in}$. lithium wire and $30 \mathrm{ml}$ of anhydrous ether. To this mixture was added $5 \mathrm{ml}$ of a solution of $18.3 \mathrm{~g}$ ( 0.134 mole) of 1-bromobutane in $25 \mathrm{ml}$ of anhydrous ether at $25{ }^{\circ} \mathrm{C}$. Gentle refluxing initiated the reaction, and the remainder of the solution of 1-bromobutane was added dropwise at $-10^{\circ} \mathrm{C}$. After the addition was completed, the reaction mixture was stirred for an additional $1 \mathrm{hr}$ at this temperature and finally allowed to warm to room temperature. The mixture was filtered, under helium, directly into a dropping funnel, and was slowly added to a solution of $25.3 \mathrm{~g}(0.134$ mole $)$ of hexafluorobenzene in $25 \mathrm{ml}$ of anhydrous ether at $-10{ }^{\circ} \mathrm{C}$. The mixture was allowed to warm slowly to room temperature, refluxed for $90 \mathrm{~min}$, and cooled in ice, and $100 \mathrm{ml}$ of ice water added. It was then filtered and the ethereal laver was separated, dried (sodium sulfate), evaporated, and distilled. After removal of $10.5 \mathrm{~g}(30 \%)$ of unreacted hexafluorobenzene, there was obtained $7 \mathrm{~g}(33.2 \%$ based on reacted hexafluorobenzene) of butyl-2,3,4,5,6-pentafluorobenzene; bp 86 to $87{ }^{\circ} \mathrm{C} / 25 \mathrm{~mm} ; \mathrm{n}_{\mathrm{D}}^{20} 1.4229$.

Analysis: Calculated for $\mathrm{C}_{10} \mathrm{H}_{9} \mathrm{~F}_{5}$ : C, 53.31; H, 4.02. Found: $\mathrm{C}, 54.0 ; \mathrm{H}, 4.2$. There was also obtained $2.5 \mathrm{~g}(11 \%)$ of a compound, bp $230^{\circ} \mathrm{C} / 1 \mathrm{~mm}$, $\mathrm{n}_{\mathrm{D}}^{20} 1.4683$, which, from its chemical analysis, appeared to be impure tributyldifluorobenzene. 'This product was not investigated further.

Analysis: Calculated for $\mathrm{C}_{18} \mathrm{H}_{28} \mathrm{~F}_{2}: \mathrm{C}, 76.9 ; \mathrm{H}$, 10.2. Found: C, 76.5; H, 11.9 .

\section{c. 2,3,4,5,6-Pentafluorobiphenyl}

In a 1-liter, three-necked flask (equipped with a stirrer having a polytetrafluoroethylene blade, a Friedrichs condenser carrying a drying tube, and a pressure-equalizing dropping-funnel fitted with a nitrogen inlet) was placed $32.7 \mathrm{~g}(0.18$ mole $)$ of hexafluorobenzene in $150 \mathrm{ml}$ of anhydrous ether. A solution of 0.18 mole of phenyllithium in $250 \mathrm{ml}$ of ether was added dropwise under a nitrogen atmosphere, with constant stirring. After the first $25 \mathrm{ml}$ had been added, heat was applied to initiate a vigorous exothermic reaction marked by the formation of a heavy, white precipitate. The remainder of the phenyllithium was added carefully so that the refluxing was vigorous but not violent. The mixture was stirred for $24 \mathrm{hr}$ at room temperature and then treated with about $200 \mathrm{ml}$ of 10 percent aqueous hydrochloric acid to destroy excess phenyllithium and to dissolve the precipitated lithium fluoride.

The aqueous layer was separated and extracted with several portions of ether. The ether solutions were combined, washed with small portions $(10 \mathrm{ml})$ of saturated sodium bicarbonate solution until the washing remained basic and with saturated sodium chloride solution until neutral, and dried (sodium sulfate). Most of the ether was removed by evaporation at atmospheric pressure through a 16-in. Vigreux column; on cooling the concentrated solution, a white precipitate formed. This was removed by filtration and was shown by nuclear magnetic resonance to be 2,3,5,6-tetrafluoro- $p$-terphenyl, $\mathrm{mp}$ $220{ }^{\circ} \mathrm{C}$. Yield: $8.5 \mathrm{~g}(17 \%)$.

Analysis: Calculated for $\mathrm{C}_{18} \mathrm{H}_{10} \mathrm{~F}_{4}$ : C, 71.5; H, 3.3 ; F, 25.1. Found: C, 68.3; H, 3.2; F, 24.7.

The filtrate was evaporated under Vigreux column to remove the residual ether. Sublimation of the residue gave white crystals which appeared to be impure 2,3,4,5,6-pentafluorobiphenyl. Yield: $33 \mathrm{~g}$ $(70 \%) ; \operatorname{mp~} 69{ }^{\circ} \mathrm{C}$.

Analysis: Calculated for $\mathrm{C}_{12} \mathrm{H}_{5} \mathrm{~F}_{5}$ : C, $59.0 \mathrm{H}, 2.1$; F, 38.9. Found: C, 59.5; H, 2.4; F, 37.5.

\section{d. 2,3,4,5,6-Pentafluoro- $\alpha$-methylstyrene}

In a 1-liter, three-necked vessel (equipped similarly to the flask used in the addition of phenyllithium) was placed a solution of $18.6 \mathrm{~g}$ (0.1 mole) of hexafluorobenzene in $100 \mathrm{ml}$ of anhydrous ether. A solution of isopropenyllithium in $250 \mathrm{ml}$ of ether (prepared from $12.1 \mathrm{~g}$ of purified 2-bromopropene by the method of Braude and Evans [57]) was transferred under helium to the dropping funnel and added dropwise to the hexafluorobenzene solution. After $25 \mathrm{ml}$ of reagent had been added, heat was applied to initiate refluxing; the solution became cloudy at once. On completing the addition of the isopropenyllithium, the mixture was refluxed for $1 \mathrm{hr}$ and then kept overnight at room temperature. After addition of about $50 \mathrm{ml}$ of 5 percent sulfuric acid, the solid was removed by filtration through a sintered-glass funnel. The ether layer of the filtrate was separated from the aqueous layer, washed with small portions of water until neutral, and dried (sodium sulfate) overnight. Fractional distillation through a 16-in. Vigreux column gave, after removal of the ether, $9 \mathrm{~g}$ of hexafluorobenzene and $5 \mathrm{~g}$ of $2,3,4,5,6$-pentafluoro- $\alpha$ methylstyrene; bp 72 to $74{ }^{\circ} \mathrm{C} / 52 \mathrm{~mm}$. Yield: 24 percent $(50 \%$ based on unrecovered hexafluorobenzene).

Analysis: Calculated for $\mathrm{C}_{9} \mathrm{H}_{5} \mathrm{~F}_{5}$ : C, 51.9; H, 2.4; F, 45.9. Found: C, 51.8; H, 2.6; F, 44.6.

About $0.1 \mathrm{~g}$ of a higher-boiling liquid was isolated. Vapor-phase, chromatographic analysis (Viton A column at $125^{\circ} \mathrm{C}$ ) of this fraction revealed the presence of two main components. The first component had the same retention time as $2,3,4,5,6$-pentafluoro$\alpha$-methylstyrene. The second component (about $50 \%$ of the fraction) had a considerably longer retention time and may possibly have been a disubstituted derivative of hexafluorobenzene. No further attempt was made to identify the higher-boiling product.

A considerable amount of a crystalline product, not readily soluble and apparently polymeric in nature, was found in the filtered solid. Hence, it appears that anionic polymerization of the olefinic products occurs quite readily under the conditions of the reaction. 


\section{e. 2,3,4,5,6-Pentafluorostyrene}

Using essentially the same type of apparatus and technique as previously described, a solution of vinyllithium (prepared from 0.1 mole of phenyllithium and 0.025 mole of tetravinyltin according to the method of Seyferth and Weiner [58]) in about $150 \mathrm{ml}$ of ether was added dropwise to $18.6 \mathrm{~g}(0.1$ mole) of hexafluorobenzene in $50 \mathrm{ml}$ of anhydrous ether. The reaction at room temperature was accompanied by precipitation of a white solid and vigorous refluxing of the solution. The flask was cooled to $0{ }^{\circ} \mathrm{C}$ about halfway through the addition, to moderate the vigorous refluxing. On completion of the addition, the mixture was kept at room temperature overnight and then refluxed for $30 \mathrm{~min}$. After being cooled, the mixture was filtered, and the filtrate was flash vacuum-distilled into a trap at $-78^{\circ} \mathrm{C}$. Some polymeric solid was left in the flask. The distillate was fractionated through a 12-in. column packed with glass helices. After removal of the ether, about $4 \mathrm{~g}$ of hexafluorobenzene was recovered. The higher-boiling residue was distilled under vacuum. A considerable quantity of solid was left in the distilling flask; it may have been polymeric vinyl derivative(s) of hexafluorobenzene. The colorless, liquid distillate, bp $34^{\circ} \mathrm{C} / 25 \mathrm{~mm}$, was shown to be identical with $2,3,4,5,6$-pentafluorostyrene obtained by dehydration of $2,3,4,5,6$-pentafluoro- $\alpha$-methylbenzyl alcohol (prepared by the reaction of pentafluorophenyl Grignard reagent with acetaldehyde [12]). Vapor-phase, chromatographic analysis showed the monomer to be of high purity. Yield: 20 percent (based on unrecovered hexafluorobenzene).

\subsection{Reaction of Hexafluorobenzene With Lithium Aluminum Hydride and Lithium Hydride}

In a $300-\mathrm{ml}$, three-necked flask equipped as described previously was placed $21 \mathrm{~g}$ (0.11 mole) of hexafluorobenzene in $50 \mathrm{ml}$ of anhydrous ether. A slurry of $3 \mathrm{~g}(0.08$ mole $)$ of lithium aluminum hydride in $200 \mathrm{ml}$ of anhydrous ether was filtered into a dropping funnel, and the filtrate was added dropwise, under a slow stream of nitrogen, to the stirred, refluxing solution of hexafluorobenzene. After all of the lithium aluminum hydride solution had been added, the mixture (containing a white precipitate) was refluxed for $8 \mathrm{hr}$. Aliquots of the mixture were periodically analyzed by vapor-phase chromatography, in order to determine the conversion of hexafluorobenzene to pentafluorobenzene as a function of time. After $1 \mathrm{hr}$, an aliquot of the ethereal solution contained two components (in addition to the ether). The component having the shorter retention time was the starting material. The other component, after isolation by preparative, vapor-phase chromatography, was shown by massspectrometric analysis to have a parent mass of 168 , corresponding to pentafluorobenzene. The conversion of hexafluorobenzene to pentafluorobenzene was estimated to be about 30 percent. After $6 \mathrm{hr}$, the conversion was about 50 percent, and, after $8 \mathrm{hr}$, 60 percent. Further refluxing did not increase the percent conversion appreciably.
The reaction mixture was cooled to $-78{ }^{\circ} \mathrm{C}$ and the excess hydrides were destroyed by adding wet ether, water, and 20-percent aqueous hydrochloric acid until the mixture was acid. The ether layer was separated, combined with the ether extracts of the water layer, and dried (sodium sulfate). The ether was removed by evaporation through a 24-in. column packed with glass-helices. The higher-boiling residue was fractionated, to give $17 \mathrm{~g}$ of a mixture of hexafluorobenzene and pentafluorobenzene, bp 78 to $84{ }^{\circ} \mathrm{C}$. Some of the hexafluorobenzene (about $3 \mathrm{~g}$ ) was apparently lost during the reaction or distillation, or both. The products were separated by preparative, vapor-phase chromatography, using a column $8 \mathrm{ft}$ by $5 / 8$ in. packed with acid-washed firebrick coated with 20 percent (by weight) of silicone oil (SE 30). The final recovery of unreacted hexafluorobenzene was $8 \mathrm{~g}$; the yield of pentafluorobenzene was $7.5 \mathrm{~g}(61 \%$, based on unrecovered hexafluorobenzene). Less than $0.5 \mathrm{~g}$ of disubstitution product (presumably 1,2,4,5-tetrafluorobenzene) was also isolated. About $1 \mathrm{~g}$ of a higher-boiling, unidentified product remained in the pot.

Several other experiments were tried, using equimolar proportions of hexafluorobenzene and lithium hydride in ether. Very little, if any, pentafluorobenzene was formed, even after long periods of reflux. The same materials were sealed in a dry, evacuated bomb, which was then heated at various temperatures ( 80 to $200{ }^{\circ} \mathrm{C}$ ) for various times (up to $24 \mathrm{hr})$. Again, no pentafluorobenzene or other product was detected by vapor-phase, chromatographic analysis [24 by 0.25 -in. column, with a packing of 30 percent (by weight) of silicone oil (Dow Corning 550) on 40-60-mesh, acid-washed firebrick] of the ether solution. However, the use of lithium aluminum hydride in 10-percent molar proportion together with equimolar proportions of lithium hydride and hexafluorobenzene in ether resulted in a 25-percent conversion to pentafluorobenzene after $48 \mathrm{hr}$ of reflux.

The authors express their appreciation and thanks to R. A. Paulson for elementary analysis, James Brown for mass-spectrometric analysis, Max Tryon for infrared spectra, and Harold Finegold for nuclear magnetic-resonance spectra.

\section{References}

[1] Y. Desirant, Bull. Acad. Roy. Belg. Classe. Sci [5] 41, $759(1955)$

[2] Y. Desirant, Bull. Soc. Chim. Belg. 67, 676 (1958).

[3] M. Hellman, E. Peters, W. J. Pummer, and L. A. Wall, J. Am. Chem. Soc. 99, 5654 (1957).

[4] J. A. Godsell, M. Stacey, and J. C. Tatlow, Nature 1\%8, 199 (1956).

[5] G. C. Finger, F. H. Reed, D. M. Burness, D. M Fort, and R. B. Blough, J. Am. Chem. Soc. 73, 145 (1951).

[6] V. H. Dibeler, R. M. Reese, and F. L. Mohler, J. Chem. Phys. 26, 304 (1957).

[7] L. A. Wall, R. E. Donadio, and W. J. Pummer, J. Am. Chem. Soc. 8\%, 4846 (1960).

[8] R. E. Florin, L. A. Wall, and D. W. Brown, J. Research NBs 64A (Phys. and Chem.) No. 4,269 (1960).

[9] M. Hellmann and A. J. Bilbo, J. Am. Chem. Soc. 75, 4590 (1953). 
[10] M. Hellmann, A. J. Bilbo, and W. J. Pummer, J. Am. Chem. Soc. $\mathbf{7 \% ,} 3650$ (1955).

[11] W. J. Pummer, R. E. Florin, and L. A. Wall, J. Research NBS 62, 113 (1959) RP 2939

[12] E. Nield, R. Stephens, and J. C. Tatlow, J. Chem. Soc. $166(1959)$

[13] M. Szware (private communication).

[14] A. L. Rocklin, J. Org. Chem. 21, 1478 (1956).

[15] M. Liveris and J. Miller, Chem. Ind. 954 (1957).

[16] J. F. Bunnett, Quart. Rev. 12, 4 (1958).

[17] R. E. Florin, W. J. Pummer, and L. A. Wall, J. Research NBS 62, 119 (1959) RP 2940.

[18] W. J. Pummer and L. A. Wall, Science 12\%, 643 (1958).

[19] J. M. Birchall and R. N. Haszeldine, J. Chem. Soc. 13 (1959).

[20] E. J. Forbes, R. D. Richardson, M. Stacey, and J. C. Tatlow, J. Chem. Soc. $\mathbf{2 0 1 9}$ (1959).

[21] E. Nield and J. C. Tatlow, Tetrahedron 8, 38 (1960)

[22] K. Wallenfels and W. Draber, Chem. Ber. 90, 2819 (1957)

[23] E. J. Forbes, R. D. Richardson, and J. C. Tatlow, Chem. Ind. 630 (1958).

24] J. E. Fearn and L. A. Wall (unpublished results).

[25] G. M. Brooke, J. Burdon, M. Stacey, and J. C. Tatlow, J. Chem. Soc. 1768 (1960).

[26] G. M. Brooke, J. Burdon, and J. C. Tatlow, J. Chem. Soc. 802 (1961).

[27] G. M. Brooke, J. Burdon, and J. C. Tatlow, Chem. Ind 832 (1961)

[28] K. H. Saunders, The aromatic diazo compounds, pp. 141, 158 (Edward Arnold and Co., London, 1949)

[29] W. J. Pummer and L. A. Wall, J. Research NBS 63A, (Phys. and Chem.) No. 2, 167 (1959).

[30] K. Wiechert, Newer methods of preparative organic chemistry p. 336 (Interscience Publishers Inc., New York, 1947).

[31] J. H. Boyer and S. E. Ellzey, Jr., J. Org. Chem. 24, 2038 (1959).

[32] W. D. Emmons, J. Am. Chem. Soc. 79, 5528 (1957).

[33] W. J. Mijs, S. E. Hoekstra, R. M. Ulmann, and E. Haringa, Rec. Trav. Chim. $\mathbf{g}, 746$ (1958)

[34] R. R. Holmes and R. P. Boyer, J. Am. Chem. Soc. 82, $3454(1960)$.
[35] W. J. Pummer and L. A. Wall, Abstracts Papers Am. Chem. Soc. 136, 24M (1959).

[36] J. M. Antonucci and L. A. Wall, Abstracts Papers Am. Chem. Soc. 140, 25 M (1961).

[37] A. K. Barbour, M. W. Buxton, P. L. Coe, R. Stephens, and J. C. Tatlow, J. Chem. Soc. 808 (1961).

[38] M. A. Doran and R. Waack, Chem. Ind. 1165 (1961).

[39] J. M. Birchall and R. N. Haszeldine, J. Chem. Soc. 3719 (1961).

[40] J. E. Johnson, R. H. Blizzard, and H. W. Carhart, J. Am. Chem. Soc. 70, 3664 (1948).

[41] J. C. Tatlow (private communication).

[42] J. F. Bunnett, Quart. Rev. 12, 1 (1958).

[43] J. F. Bunnett, Kekule symposium, p. 144 (Butterworths, London, 1959).

[44] R. Huisgen, Kekule symposium, p. 158 (Butterworths, London, 1959).

[45] A. J. Parker, Quart. Rev. 16, 163 (1962).

[46] P. L. Coe, R. Stephens, and J. C. Tatlow, J. Chem. Soc. 322\% (1962).

[47] C. R. Patrick and G. S. Prosser, Nature 18\%, 1021 (1960).

[48] D. M. G. Lawrey and H. MeConnell, J. Am. Chem. Soc. \%4, $6175(1952)$

[49] A. W. Baker, J. Am. Chem. Soc. 80, 3598 (1958).

[50] A. W. Baker and W. W. Kaeding, J. Am. Chem. Soc. 81, $5904(1959)$

[51] T. Ri and H. Eyring, J. Chem. Phys. 8, 433 (1940).

[52] C. C. Meredith and G. Wright, Can. J. Chem. 38, 1177 (1960).

[53] C. K. Ingold, Structure and mechanism in organic chemistry, p. 741 Cornell Univ. Press, Ithaca, New York, 1953).

[54] G. M. Brooke, J. Burdon, and J. C. Tatlow, J. Chem. Soc. 3253 (1962).

[55] P. Robson, M. Stacey, R. S. Stephens, and J. C. Tatlow, J. Chem. Soc. 4754 (1960)

[56] A. I. Vogel, Practical organic chemistry, p. 188 (Longmans, Green and Co., Inc., New York, 1954).

[57] E. A. Braude and E. A. Evans, J. Chem. Soc. 333 (1956).

[58] D. Seyferth and M. A. Weiner, Chem. Ind. 402 (1959).

(Paper 67A5-237) 


\title{
Publications of the National Bureau of Standards ${ }^{\star}$
}

\author{
Selected Äbstracts
}

Standard X-ray diffraction powder patterns, H. E. Swanson, M. C. Morris, R. P. Stinchfield, and E. H. Evans, NBS Mono. 25, Section 2 (May 3, 1963), 35 cents.

Standard X-ray diffraction powder patterns are presented for the following thirty-seven substances: $\mathrm{Al}\left(\mathrm{PO}_{3}\right)_{3}, \mathrm{SbF}_{3}$, $\mathrm{Ba}_{3}\left(\mathrm{AsO}_{4}\right)_{2}$ *, $\mathrm{Ba}\left(\mathrm{ClO}_{4}\right)_{2} \cdot 3 \mathrm{H}_{2} \mathrm{O}, \mathrm{Cd}(\mathrm{CN})_{2} *, \mathrm{CdWO}, \mathrm{Cs}_{2} \mathrm{OsBr}_{6}$ *, $\mathrm{Cs}_{2} \mathrm{OsCl}_{6} *, \quad \alpha-\mathrm{CrPO}_{4}, \quad \mathrm{Co}\left[\mathrm{Hg}(\mathrm{CNS})_{4}\right]^{*}, \quad \beta-\mathrm{CoSO}_{4}, \quad \mathrm{Dy}_{3} \mathrm{Ga}_{2}$ $\left(\mathrm{GaO}_{4}\right)_{3} *, \mathrm{ErMnO}_{3} *, \mathrm{Eu}_{3} \mathrm{Ga}_{2}\left(\mathrm{GaO}_{4}\right)_{3} *, \mathrm{Gd}_{3} \mathrm{Ga}_{2}\left(\mathrm{GaO}_{4}\right)_{3} *$, $\mathrm{Li}_{3} \mathrm{AsO}_{4} *, \mathrm{Li}_{3} \mathrm{P}_{3} \mathrm{O}_{9} \cdot 3 \mathrm{H}_{2} \mathrm{O} *, \mathrm{Li}_{2} \mathrm{WO}_{4} \cdot \frac{1}{2} \mathrm{H}_{2} \mathrm{O} *, \mathrm{Lu}_{3} \mathrm{Ga}_{2}\left(\mathrm{GaO}_{4}\right)_{3}$ *, $\mathrm{LuMnO}_{3} *, \mathrm{MnWO}_{4}$ (huebnerite), $\mathrm{HgF}_{2}$ *, $\mathrm{NiSO}_{4}, \mathrm{NiWO}_{4}$ *, $\mathrm{K}_{2} \mathrm{ReCl}_{6}$, $\mathrm{K}_{2} \mathrm{RuCl}_{5} \mathrm{NO}^{*}, \mathrm{RbClO}_{4} * \mathrm{RbIO}_{4} * \mathrm{Ag}_{2} \mathrm{SeO}_{4} *$, $\mathrm{NaCNO}^{*}, \quad \mathrm{Na}_{2} \mathrm{WO}_{4} \cdot 2 \mathrm{H}_{2} \mathrm{O}, \quad \beta-\mathrm{Na}_{4} \mathrm{P}_{4} \mathrm{O}_{12} \cdot 4 \mathrm{H}_{2} \mathrm{O}, \quad \mathrm{Sr}_{3}\left(\mathrm{AsO}_{4}\right)_{2} *$, $\mathrm{Tl}_{3} \mathrm{AsO}_{4} *$, $\mathrm{TlClO}_{4} *, \mathrm{YAsO}_{4}, \mathrm{ZnWO}_{4} *$. Eleven are to replace patterns already given in the X-ray Powder Data File issued by the American Society for Testing and Materials, and twenty-six patterns indicated by asterisks are for substances not previously included. The X-ray Powder Data File is a compilation of diffraction patterns from many sources and is used for the identification of unknown crystalline materials by matching spacing and intensity measurements. The patterns were made with a Geiger counter X-ray diffractometer using samples of high purity. When possible, the $d$-values were assigned Miller indices determined by comparison with calculated interplanar spacings and from space group extinctions. The densities and lattice constants were calculated, and the refractive indices were measured whenever possible.

Mechanical behavior of crystalline solids (Proceedings of an American Ceramic Society Symposium, New York City, A pril 1962), NBS Mono. 59 (Mar. 25, 1963), \$1.75.

This Monograph represents the Proceedings of a Symposium on The Mechanical Behavior of Crystalline Solids, held under the auspices of the Ceramic Educational Council of the American Ceramic Society, with the cooperation of the National Bureau of Standards, and under the sponsorship of the Edward Orton Junior Ceramic Foundation, and the Office of Naval Research. The Symposium took place at the 64th Annual Meeting of the American Ceramic Society, in New York, on April 28 and 29, 1962.

Testing of metal volumetric standards, J. C. Hughes and B. C. Keysar, NBS Mono. 62 (Apr. 1, 1963), 15 cents.

The National Bureau of Standards has for many years calibrated and certified metal measures which are used as standards by weights and measures officials and others in the calibration of instruments for measuring the volumes of fluids. No complete specifications or tolerances for these standards have ever been published, however, nor have standardized procedures for the calibration and use of the liquid measures been available.

The information contained in this Monograph should assist in the purchase of quality instruments and the proper use of the standards in calibrating other measures for liquids and gases.

- Radiobiological dosimetry, Recommendations of the International Commission on radiological units and measurements, NBS Handb. 88 (A pril 30, 1963), Supersedes parts of Handb. 78 . Handbooks 84 through 89 will completely replace Hy8, 25 cents.

This publication deals primarily with radiobiological dosimetry, and considers methods of improving the accuracy and intercomparability of absorbed dose measurements in radiobiology. It is in effect a handbook for the experimental radiobiologists. It emphasizes the great importance of planning the experimental work in a way which makes the dosimetry easier and more accurate and it illustrates how this can be done.
The publication was prepared by a committee of the International Commission on Radiological Units and Measurements.

Transistorized building blocks for data instrumentation, R. L. Hill, NBS Tech. Note 168 (Apr. 1, 1963), 55 cents.

The National Bureau of Standards has developed a number of modular transistorized digital circuits that have been used in automatizing many data recording and preliminary processing tasks encountered in its scientific operations. These versatile building blocks can be connected together systematically to form digital circuits that accept raw data from experimental equipment and transpose these data into a form suitable for input to a high-speed electronic computer.

Each assembly of packages can be tailored to fit the special requirements of the project and can be used at the site of the experiment. The output from the system can be: 1) fed directly to a computer, 2) recorded on a medium (paper tape, magnetic tape, etc.) suitable for computer input at a later date, or 3 ) used to drive display equipment that keeps the scientist informed of the progress of his experiment.

As a result of experience in the application of these units, some of the original packages have been modified and additional types developed. In addition to describing the modified and new package types, this report also includes a description of a new series of packages consisting of identical circuitry, but utilizing a different type of mating connector and a smaller circuit-board.

Practical methods for calibration of potentiometers, ${ }^{\prime}$ D Ramaley, NBS Tech. Note 172 (Mar. 25, 1963), 30 cents. Potentiometer circuitry, particularly as related to calibration, is discussed with the primary consideration given to the required circuit measurements. The more feasible means of calibrating potentiometers are described in considerable detail. Emphasis is placed upon the use of the Universal Ratio Set as the basic implement for accomplishing the major portion of potentiometer calibrations.

Tables to facilitate the determination of the ferrimagnetic resonance linewidth of non-metallic magnetic materials, C.C. Preston and W. E. Case, NBS Tech. Note 173 (A pr. 15, 1963), 25 cents.

A common test procedure for measuring ferrimagnetic resonance linewidth, $\Delta H$, and gyromagnetic ratio, $\gamma$, of microwave ferrites is based upon a perturbation analysis of the complex frequency shift obtained when a small sample is placed in a resonant cavity and an applied d-c magnetic field. However, this method necessitates the plotting of a ferrimagnetic resonance loss curve. To find $\Delta H$ it is possible to derive an equation using lumped circuit theory which reduces the number of needed measurements to four attenuator readings.

This paper provides a table which gives values of attenuation according to the following formula which facilitates the determination of linewidth:

$$
\begin{gathered}
A=20 \log 2-20 \log \left(10 \frac{A_{0}-A_{r}}{20}+1\right) \text { for } A_{0}-A_{r} \\
=0.41(0.01) 32.40 .
\end{gathered}
$$

Calibration of volt-ampere converters, E. S. Williams, NBS Tech. Note 188 (A pr. 25, 1963), 20 cents.

These notes have been prepared to describe the National Bureau of Standards calibration services for volt-ampere converters (or transfer volt-ammeters), to suggest procedures for d-c standardization in the user's laboratory, and to de- 
scribe a voltage comparator which can be used to make such calibrations quickly and easily.

Degradation of polymers, L. A. Wall and J. H. Flynn, Rubber Chem. Technol. XXXV, No. 5, 1157-1221 (December 1962).

Recent literature pertaining to both the theoretical aspects and experimental results of the degradation of polymers by heat and radiation is reviewed and critically evaluated. Theories of random and chain thermal degradation of vinyl polymers and copolymers are reviewed. The degradation of polymethacrylates, polyacrylates, poly- $\alpha$-methylstyrene, polyolefins, polystyrene, other vinyl polymers, cellulose, polyesters, polyamides, dienes, natural rubber, and copolymers are discussed in the light of these theories. The thermodynamics and energetics of the degradation of these polymers is also reviewed.

Chain scission, crosslinking, and gel formation and the kinetic mechanisms of these processes which take place during degradation of polymers by ionizing radiation and ultraviolet light are included. Degradative, rather than synthetic, effects are emphasized in the discussion.

Chemical and magnetic enhancement of perturbed lines in the violet spectrum of $\mathbf{C N}, H$. E. Radford and $H$. P. Broida, J. Chem. Phys. 38, No. 3, 644-65\% (Feb. 1, 1963).

A chemical kinetic theory based on the quantum mechanical properties of perturbed molecular states is developed to explain the rotational intensity anomalies observed in the CN band spectrum emitted by an active nitrogen flame. Relative intensities of perturbed lines are calculated in terms of parameters which specify the rates of chemical formation, collisional relaxation, and radiative decay of $\mathrm{CN}$ molecules in the excited electronic states where the perturbed lines originate. Numerical values of these parameters are found which, inserted in the intensity expressions, predict correctly the observed pressure-dependent enhancement of each perturbed line. From this analysis the approximate value $6 \times 10^{-7} \mathrm{sec}$ is found for the radiative lifetime of the $\mathrm{A}^{2} \Pi_{\mathrm{i}}$, $\mathrm{v}^{\prime}=10$ state of $\mathrm{CN}$. It is also found that nearly every gas kinetic collison changes the rotational state of an excited CN molecule, but that only about 1 collision in 100 can cause the exchange of vibrational energy for electronic energy represented by the transition $\mathrm{A}^{2} \Pi_{i}, \mathrm{v}^{\prime}=10 \rightarrow \mathrm{B}^{2} \Sigma^{+}, \mathrm{v}^{\prime}=0$. The theory also predicts, in agreement with recent observations, an additional selective enhancement of certain perturbed lines by a strong magnetic field.

Profiles of Stark-broadened Balmer lines in a hydrogen plasma, W. L. Wiese, D. R. Paquette, and J. E. Solarski, Phys. Rev. 129, No. 3, 1225-1232 (Feb. 1, 1963).

A stationary hydrogen plasma was generated in a high current stabilized arc and its temperature was determined spectroscopically from the diagnostics of the line and continuum radiation. Extensive measurements of the profiles of the Balmer lines $\mathrm{H}_{\beta}$ and $\mathrm{H}_{\gamma}$ where made and show that recent improvements in the theory of Stark broadening described the observed line shapes well. Electron densities determined from line width measurements are slightly higher than those derived from temperature measurements. In the covered range of electron densities from $10^{16}-10^{17} \mathrm{~cm}^{-3}$ and temperatures arond $10^{4}{ }^{\circ} \mathrm{K}$ the difference amounts to $25 \%$ for $\mathrm{H}_{\gamma}$ and $12 \%$ for $\mathrm{H}_{\beta}$, for which a refined Stark broadening theory is available. In addition, the intensity distribution in the distant linewings of $\mathrm{H} \alpha$ and $\mathrm{H} \beta$ was measured and compared with asymptotic formulae. Satisfactory agreement is obtained.

Magnetic susceptibilities and dilution effect in low-spin $\mathrm{d}^{4}$ complexes: Osmium (IV), R. B. Johannesen and G. A. Candela, Inorg. Chem. 2, 67-72 (1963).

Magnetic susceptibility measurements were made on a series of hexachloro- and hexabromoosmates, $\mathrm{M}^{2} \mathrm{OsX}_{6}$, and on potassium hexachlororuthenate, $\mathrm{K}_{2} \mathrm{RuCl}_{6}$. The paramagnetism of the Os (IV) complexes is independent of temperature and increases as the osmium ions are separated. It is shown that the effect of dilution is described by the equation

$$
\chi=\frac{\chi_{\infty}}{1+\lambda d^{-\mathrm{n}} \chi_{\infty}}
$$

where $\chi_{\infty}$ is the susceptibility at infinite dilution, $d$ is the average distance between the osmium ions, $\lambda$ is a constant, and $\mathrm{n}$ is approximately 2 .

An intermediate coupling scheme is necessary to explain the experimental results for the $5 d^{4}$ configuration. In this scheme the only parameters are the spin-orbit coupling constant and the orbital reduction factor. The values of $2300 \pm 300 \mathrm{~cm}^{1}$ for the spin-orbit coupling constant and $3500 \pm 200 \mathrm{~cm}^{-1}$ for the Coulomb interaction coefficient were calculated.

New absolute null method for the measurement of magnetic susceptibilities in weak low-frequency fields, C. T. Zahn, Rev. Sci. Instr. 34, No. 3, 285-291 (Mar. 1963).

Use is made of the magnetic equivalence of a uniformly polarized volume of paramagnetic material and a solenoid carrying electric current, to design a permanent variable standard of magnetic susceptibility. Such a standard is incorporated into a magnetic susceptibility bridge in a simple manner, surrounding the specimen; and the bridge is thereby transformed into an absolute null instrument of high accuracy and sensitivity, and of great ease and low cost of construction and operation. By this method numerous particular advantages of other methods are combined; and some of their notable limitations are overcome. A preliminary application was made showing that the bridge performs as expected. Important features in the design of this bridge are discussed. A detailed consideration of sources of error suggests that it may eventually be possible by this method to obtain greater absolute accuracy than by other known methods.

A method for measuring the instability of resistance strain gages at elevated temperatures, R. L. Bloss and J. T. Trumbo, ISA Trans. 2, No. 2, 112-116 (A pr. 1963).

The usefulness of resistance strain gages at elevated temperatures is frequently limited by the instability of gage resistance with time. Methods and equipment that have been developed to measure this effect are described.

Intercomparison of national roentgen and gamma ray exposure-dose standards, H. O. Wyckoff, A. Allisy, G. H. Aston, G. P. Barnard, W. Hubner, T. Loftus, and G. Taupin, Acta Radiol. 1, No. 1, 5\%-78 (Feb. 1963).

Indirect intercomparisons of calibrations of ionization chambers for exposure dose measurements in roentgens have been completed between the national standards of France, Germany, U.K., and U.S.A., with an estimated maximum uncertainty of about 0.5 percent. This is somewhat larger than that assigned to direct comparisons (Aston and Attix, 1957). Several revisions in the calibration procedure are indicated which may reduce the uncertainty in the indirect intercomparisons.

The present calibrations agree with those obtained earlier between three of the laboratories to within the expected uncertainty, 0.5 percent; also the calibrations at PTB agree with those at NBS to within 0.6 percent. However the results from NPL and PTB disagree by slightly more than one percent for nearly the same quality of the harder radiations. A detailed redetermination of some of the correction factors may be needed to remove this discrepancy.

Wavelengths, energy levels, and pressure shifts in mercury 198, V. Kaufman, J. Opt. Soc. Am. 52, No. 8, 866-870 (Aug. 1962).

The vacuum wavelengths of 27 lines of $\mathrm{Hg}^{198}$ and 6 lines of $\mathrm{Kr}^{86}$ have been measured relative to the international standard of length, the $\mathrm{Kr}^{86}$ line at $6057.80211 \AA$, by photographic Fabry-Perot interferometry. These measurements were made with $\mathrm{Hg}^{198}$ electrodeless lamps containing argon at pressures of $1 / 4,3$, and $10 \mathrm{~mm} \mathrm{Hg}$ and a $\mathrm{Kr}^{86}$ hot-cathode lamp operated according to the conditions prescribed by the International Conference on Weights and Measures in 1960 Energy-level values have been derived from the wavelengths of each of the $\mathrm{Hg}^{198}$ sources, and on the basis of these values, the energy level and wavelength shifts per unit pressure of argon have been calculated. The suitability of the $\mathrm{Hg}^{198}$ electrodeless lamp as a source of wavelength standards for interferometric measurement of length and wavelength is discussed.

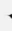

s

$\leftarrow$

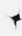

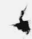
$\checkmark$ 
Audio-írequency compliances of prestressed quartz, fused silica, and aluminum, M. Greenspan and C. Tschiegg, Proc. Fourth Intern. Congr. on Acoustics, Part I, Paper P12 (Copenhagen, Denmark, Aug. 21-28, 1962).

An attempt was made to find the excess compliances associated with dispersions found by Fitzgerald. Compliances were obtained from resonant frequencies of fixed-free composite reeds. Prestress was either piezoelectrically or thermally induced. No excess compliances were observed.

X-ray microscopy of polymers by point projection, S. B. Newman, Mod. Plastics 40, No. 7, 165-179 (Mar. 1963). A two-lens electromagnetic microfocus X-ray tube has been used for studying the microstructure of a number of polymeric materials. Long wavelength radiation from aluminum targets was used in most of the work, though copper targets were used in a few experiments. Results obtained from foams, laminates, fibers, and fabrics are presented. The potential of available instrumentation for application to this genera! material area is considered.

Microwave spectrum and structure of difluoramine, D. R. Lide, Jr., J. Chem. Phys. 38, No. 2, 456-460 (Jan. 15, 1963). The microwave spectra of $\mathrm{NF}_{2} \mathrm{H}$ and $\mathrm{NF}_{2} \mathrm{D}$ have been analyzed. Rotational constants are 53017.12, 10895.43, 9307.22 Mc for $\mathrm{NF}_{2} \mathrm{H}$ and 44884.92, 10749.38, 9126.26 Mc for $\mathrm{NF}_{2} \mathrm{D}$. The structure obtained from these constants is: $\mathrm{r}_{\mathrm{NF}}=1.400 \pm 0.002 \mathrm{~A}, \mathrm{r}_{\mathrm{NH}}=1.026 \pm 0.002 \mathrm{~A}, \angle \mathrm{FNF}=102.9^{\circ}$ $\pm 0.2^{\circ}$, and $\angle \mathrm{HNF}=99.8^{\circ} \pm 0.2^{\circ}$. Quadrupole coupling constants have been measured for both isotopic species and the complete field-gradient tenser has been determined. The dipole moment has a magnitude of $1.93+0.02 \mathrm{D}$ and makes an angle of $19^{\circ}$ with the NH bond. An upper limit of $0.2 \mathrm{Mc}$ may be set on the inversion splitting in the ground state.

Design of low voltage electron guns, J. A. Simpson and C. E. Kuyatt, Rev. Sci. Instr. 34, No. 3, 265-268 (Mar. 1963).

It is shown that by use of a multistage technique in which electrons are drawn from a cathode by a high potential and decelerated to the required final energy, guns can be designed capable of forming beams in which the current is limited only by space charge in the beam itself. The design principles and procedures are given and illustrated by two examples of electron guns giving highly collimated beams and operating at energies of 30 and $500 \mathrm{eV}$. The measured currents obtained are somewhat greater than the space charge limited beam maximum because of ion neutralization.

Determination of source self-absorption in the standardization of electron-capturing radionuclides, S. B. Garfinkel and J. M. R. Hutchinson, Intern. J. Appl. Radiation and Isotopes 13, 629-689 (1962).

A method is described for measuring source self-absorption in the standardization of radionuclides which decay by electron capture directly to the ground state. Auger electrons are used as a tracer in order to determine the $\mathrm{X}$-ray absorption. Data are presented which indicate that X-ray absorption in a certain type of electroplated source is less than $0.2 \%$. A method for determination of ejected photoelectrons from absorbing foils in $4 \pi$ X-ray counting is described.

Mean first-passage times and the dissociation of diatomic molecules, K. E. Shuler and G. H. Weiss, J. Chem. Phys. 38, No. 2, 505-509 (Jan. 15, 1963).

The collisional activation of diatomic molecules to dissociation can be considered as a random walk with an absorbing barrier in the energy space of the molecules. In such a model, the rate of activation to dissociation is related to the mean first passage time of the molecules across the absorbing barrier. We have computed here the mean first passage time for an ensemble of oscillators in an initial Boltzmann distribution with transitions limited to nearest neighbor states $(|\Delta \nu|=1)$ and for an ensemble of oscillators initially in the ground state $(\nu=0)$ which undergo transitions between non-nearest neighbor states $(|\Delta \nu| \geq 1)$. It is shown that the magnitude of the mean first passage time is very sensitive to the height (in energy space) of the absorbing barrier and to the "jump distance", $|\Delta \nu|>1$, for non-nearest neighbor transitions. The implications of these results on the mechanism of the activation and dissociation of diatoms are discussed briefly.

Infrared absorption spectra of carbon suboxide and malonotitrile in solid argon matrices, L. L. Ames, D. White, and D. E. Mann, J. Chem. Phys. 38, No. 4, 910-917 (Feb. 15, 1.963). The infrared spectra of carbon suboxide and malononitrile isolated in solid argon matrices at low temperatures have been measured in the spectral region $440-4400 \mathrm{~cm}^{-1}$. A detailed comparison with prior spectroscopic data is made. It is shown that the matrix spectra of $\mathrm{C}_{3} \mathrm{O}_{2}$ support the linear symmetric model. A comparison of the matrix spectra and vibrational assignments of the three isoelectronic molecules $\mathrm{C}_{3} \mathrm{O}_{2}, \mathrm{CH}_{2}(\mathrm{CH})_{2}$, and $\mathrm{B}_{2} \mathrm{O}_{3}$ is shown to favor a large apex angle in $\mathrm{B}_{2} \mathrm{O}_{3}$.

Interaction matrix element in a shell model, U. Fano, ${ }^{\mathrm{T}} \mathrm{F}$. Prats, and Z. Goldsmith, Phys. Rev. 129, No. 9, 2643-2652 (Mar. 16, 1963)

The matrix elements of two-particle interactions between states of many-particle configurations are expressed as products of one-particle reduced matrix elements and of a single recoupling coefficient. Applications are given to the Coulomb interaction of $l^{n} l^{\prime}$ configurations and to all three-electron configurations.

Electron impact ionization of atomic hydrogen, S. Geltman, M. R. H. Rudge, and M. J. Seaton, Proc. Phys. Soc. 81, Pt. 2, No. 520, 375-378 (1963).

The cross section for ionization of atomic hydrogen is calculated by several methods, including the Born-Oppenheimer and Born-Exchange Approximation, and the resulting curves compared with experiment.

Effective diffusion constant in a polyelectrolyte solution J. L. Jackson and S. R. Coriell, J. Chem. Phys. 38, No. 4, 959-968 (Feb. 15, 1962).

A general theory of the effective diffusion constant of ions in a periodic electric field is presented. The predictions of the theory are compared with the results of experiments on the diffusion of labeled sodium in a sodium hydroxidepolyacrylic acid solution.

Electron attachment coefficients of some hydrocarbon flame inhibitors, T. G. Lee, J. Phys. Chem. 67, 360-366 (1963). The electron attachment coefficients of a number of halogenated hydrocarbons, particularly flame inhibitors, were determined as a function of the average agitation energy of electrons between 0.1 and $1.2 \mathrm{ev}$ at atmospheric pressure and room temperature. A sensitive electron swarm technique was used for the measurement. The gas under study was introduced, at low concentrations, by means of a nitrogen carrier into an ionization chamber.

The electron attachment coefficients for the compounds examined range from $1 \times 10^{-5}$ to 10 for $\mathrm{CF}_{4}$ and $\mathrm{CF}_{2} \mathrm{Br}_{2}$, respectively. All inhibitors studied were found to have a higher electron attachment coefficient than $\mathrm{O}_{2}$. It was found, with some exceptions, that gases reported as effective hydrocarbon flame inhibitors exhibited high electron attachment coefficients.

The measurement of moisture boundary layers and leaf transpiration with a microwave refractometer, D. M. Gates, M. J. Vetter, and M. C. Thompson, Jr., Nature 197, $10 \% 0^{-}$ 1072 (Mar. 16, 1963).

A microwave refractometer has been used as a hygrometer to measure the moisture gradient found near a free water surface and near the surface of a leaf. Interesting transpiration effects were observed for begonia and bean leaves when the leaves were stimulated with light. The instrument samples the air through a small orifice and thereby produces very little disturbance to the moisture boundary layer under investigation.

The history of $\mathbf{P t} \mathbf{2 7}$, E. Wichers, Book, Temperature, Its Measurement and Control in Science and Industry, 3, Pt. 1, 259-262 (Reinhold Publ. Corp, New York, N.Y., 1962).

This is a brief account of work done at the National Bureau of Standards in the period 1919-22 on the preparation of platinum of high purity and the conditions required to melt 
the purified metal with minimum contamination by the crucible material. Contamination with calcium was correlated with a positive thermal emf toward purer specimens. Negative thermal emf was also found to correlate with an increasing temperature coefficient of electrical resistivity. Trial melts were identified by numbers referring to notebook descriptions of the experimental methods used. The melt numbered 27 turned out to be thermoelectrically negative to all other specimens prepared up to that time, as well as to all specimens from commercial sources. It thus came into use as a standard for further comparisons in the NBS labora. tory and later into similar use in the platinum industry.

Some causes of resonant frequency shifts in atomic beam machines. I. Shifts due to other frequencies of excitation, J. H. Shirley, J. Appl. Phys. 34, 783-788 (Apr. 1963).

The quantum theory of an atomic beam machine is set up in matrix form. A new method is then used to derive the BlochSiegert shift in resonance. The results are extended to the case of Ramsey type excitation. Finally the Bloch-Siegert shift is computed for the present atomic beam frequency standards and found to be well below the accuracy of measurement.

Some causes of resonant frequency shifts in atomic beam machines. II. The effect of slow frequency modulation on the Ramsey line shape, J. H. Shirley. J. Appl. Phys. 34, 789791 (A pr. 1963).

The effect of slow frequency modulation of the exciting radiation on the Ramsey line shape observed in an atomic beam experiment is formulated theoretically. It is shown that the presence of second harmonic in the modulation can introduce measurable frequency shifts, whether observed directly or with a servo-system.

The specific heat at constant volume of parahydrogen at temperatures from 15 to $90^{\circ} \mathrm{K}$ and pressures to $340 \mathrm{~atm}$, B. A. Younglove and D. E. Diller, Cryogenics 2, No. 6, 1-5 (Dec. 1962).

The specific heats at constant volume of parahydrogen have been measured at temperatures from 15 to $90^{\circ} \mathrm{K}$ and at densities from 0.7 to 2.6 times the critical density. This paper describes the experimental method, the analysis and reduction of the data, and presents the results in the form of tables and graphs.

Electron spin resonance of gamma-irradiated cellulose, R. E. Florin and L. A. Wall, J. Polymer Sci. 1, Pt. A, 1163-117s (1963).

Irradiation of purified cellulose with cobalt-60 gamma ravs, in vacuo, produced moderate yields of radicals detectable by electron spin resonance. The ESR spectrum is asymmetric, with five partially resolved peaks occupying a total region about 50 gauss wide, for spectroscopic splitting factor near $\mathrm{g}=2$. The peaks show differing saturation behavior with increasing microwave power. Differences of crystallinity have no obvious effect on the yield or nature of the radical ESR spectrum. The presence of about $8 \%$ water during irradiation decreases the yield greatly and modifies the hyperfine structure. For dry cellulose the initial G-value for radicals is near 2.8 radicals per 100 e.v. absorbed, but the concentration levels off at doses a little over $6 \times 10^{20} \mathrm{ev} / \mathrm{g}$. Despite the early falling-off in yield, the radicals in stored irradiated specimens do not decay appreciably in several days. Even upon opening to the air the decay is quite slow. Thermal decay is imperfectly second-order, fairly rapid above $120^{\circ} \mathrm{C}$, and indicates several radicals of differing lifetime.

The speed of light, A. G. MeNish, IRE. Trans. Instr. I-11, Nos. $3 \& 4$, 138-148 (Dec. 1962).

Numerous measurements of the speed of light published during the last 30 years lead to widely divergent results as compared with the assigned experimental uncertainties. Because of wide diversity in the methods employed in the measurements, all of the data may not be combined effectively in a grand averaoe. Sufficient data had been obtained by the geodimeter method to group them and derive a statistical estimate of the uncertainty in the speed of light by this method. This result, and conclusions reached from careful examination of several experiments, leads to the conclusion that the value $299,792.5 \mathrm{~km}$ per sec which has been internationally adopted for use in radio propagation and geodetic work is very close to the best value and not likely to be in error by as much as one part in one million.

Optimum estimators of the parameters of negative exponential distributions from one or two order statistics, M. M. Siddiqui, Ann. Math. Stat. 34, 117-121 (Mar. 1963).

Optimum estimators for the parameters of negative exponential populations from one or two order statistics are obtained analytically, thus providing solutions for all sample sizes to the problems solved by Harter (Ann. Math. Stat. 32, pp. 1078-1090) numerically for samples of sizes up to 100.

Crystallographic changes with the substitution of aluminum for iron in dicalcium ferrite, D. K. Smith, Acta Cryst. 15, 1146-1152 (Jan. 1962).

Single-crystal X-ray precession patterns of several compositions in the solid solution series

$$
\mathrm{Ca}_{8}\left(\mathrm{Fe}_{1}{ }^{-} \mathrm{Al}_{p}\right)_{8} \mathrm{O}_{20}(0 \leq p \leq 0 \cdot 69)
$$

show that the series is not completely isostructural. Systematic extinctions of $\mathrm{X}$-ray reflections indicte that the space group for compositons with the ratio $p \equiv \mathrm{Al} /(\mathrm{Al}+\mathrm{Fe})$ less than 0.30 is Pnma, whereas the space group for $p$ greater than 0.33 is Imma. The cell constants vary continuously throughout the symmetry change.

The crystal structure determined by Bertaut, Blum \& Sagnières $(1957,1959)$ for the iron end-member has the 8 Fe in two 4-fold sites, one site octahedrally coordinated with oxygen and one tetrahedrally coordinated with oxygen. The layers of octahedra correspond to the space group Imma, but the layers of tetrahedra reduce the symmetry to Pnma. The substitution of $\mathrm{Al}$ for $\mathrm{Fe}$ in this structure causes the tetrahedral layer to adjust gradually to the Imma symmetry. Steric considerations and relative intensities of $0 k 0$ reflections for various compositions suggest that the $\mathrm{Al}$ atoms substitute preferentially for tetrahedrally-coordinated Fe atoms until about half the tetrahedral cations are Al. Then additional Al distributes itself nearly equally between the tetrahedral and octahedral sites until it saturates the tetrahedral sites at approximately $p=0 \cdot 69$, the composition limit for crystallization of this phase from $\mathrm{CaO}-\mathrm{Fe}_{2} \mathrm{O}_{3}-\mathrm{Al}_{2} \mathrm{O}_{3}$ melts.

\section{Other NBS Publications}

J. Research NBS 67B (Math. and Math. Phys.), No. 3 (July-Sept. 1963), 75 cents.

Remarks on hypo-elasticity. C. Truesdell.

Error bounds in the pointwise approximation of solutions of elastic plate problems. J. H. Bramble and L. E. Payne.

Effect of error in measurement of elastic constants on the solutions of problems in classical elasticity. J. H. Bramble and L. E. Payne.

Eigenfunctions of the $f^{3}$ configuration. J. C. Eisenstein.

Zeros of first derivatives of Bessel functions of the first kind, $J_{n}^{\prime}(x), 21 \leq n \leq 51,0 \leq x \leq 100$. G. W. Morgenthaler and H. Reismann.

J. Research NBS 67C (Eng. and Instr.), No. 3 (July-Sept. $1963), 75$ cents.

Apparatus for the detection of piezoelectric coupling. L. Frenkel.

Large aperture interferometers with small beam dividers. J. B. Saunders.

A far-infrared vacuum grating spectrometer. L. R. Blaine.

Relation of emittance to other optical properties. J. C. Richmond.

Minimization of the arrestment error in one-pan two-knife balance systems. H. A. Bowman and H. E. Almer.

Determination of residual thiosulfate in processed film. C. I. Pope.

Drag compensation and measurement with manned satellites: feasibility study. R. M. Langer and J. P. Vinti.

Surface flame propagation on cellulosic materials exposed to thermal radiation. D. Gross and J. J. Loftus.

Parallel reversible permeability measurement techniques from $50 \mathrm{kc} / \mathrm{s}$ to $3 \mathrm{Gc} / \mathrm{s}$. C. A. Hoer and R. D. Harrington. 
J. Research NBS 67D (Radio Prop.), No. 5 (Sept.-Oct. 1963)

\section{0 cents.}

Ionespheric VHF scattering near the magnetic equator during the International Geophysical Year. R. Cohen and K. L. Bowles.

Radio pulse propagation by a reflection process at the lower ionosphere. J. R. Johler.

Field of a horizontal magnetic dipole in the presence of a magnetoplasma halfspace. G. Tyras, A. Ishimaru, and H. M. Swarm.

Reflection of VLF radio waves from an inhomogeneous ionosphere. Part II. Perturbed exponential model. J. R. Wait and L. C. Walters.

Collisional detachment and the formation of an ionospheric $C$ region. E. T. Pierce.

Magnetic torques and Coriolis effects on a magnetically suspended rotating sphere. J. C. Keith.

Radiation field characteristics of lightning discharges in the band $1 \mathrm{kc} / \mathrm{s}$ to $100 \mathrm{kc} / \mathrm{s}$. W. L. Taylor.

Low-frequency radio propagation into a moderately rough sea. D. F. Winter.

VLF superdirective loop arrays. E. W. Seeley.

Curves of ground proximity loss for dipole antennas (a digest). L. E. Vogler and J. L. Noble.

Observations and results from the "hiss recorder," an instrument to continuously observe the VLF emissions. J. M. Watts, J. A. Koch, and R. M. Gallet.

Influence of a sector ground screen on the field of a vertical antenna, J. R. Wait and L. C. Walters, NBS Mono. 60 (Apr. 15, 1963), 25 cents.

Quarterly radio noise data, June, July, August 1962, W. Q. Crichlow, R. T. Disney, and M. A. Jenkins, NBS Tech. Note 18-15 (Mar. 1, 1963), 45 cents.

Mean electron density variations of the quiet ionosphere, November 1959 , J. W. Wright, L. R. Wescott, and D. J. Brown, NBS Tech. Note 40-9 (April 22, 1963), 35 cents.

Mean electron density variations of the quiet ionosphere, December 1959, J. W. Wright, L. R. Wescott, and D. J. Brown, NBS Tech. Note 40-10 (March 24, 1963), 35 cents.

The error rates in multiple FSK systems and the signal-tonoise characteristics of FM and PCM-FS systems, H. Akima, NBS Tech. Note 167 (Mar. 25, 1963), 40 cents.

Bibliography on atmospheric aspects of radio astronomy, including selected references to related fields, W. Nupen, NBS Tech. Note 171 (May 1, 1963), \$2.00.

Curves of ground proximity loss for dipole antennas, L. E. Vogler and J. L. Noble, NBS Tech. Note 175 (May 20, 1963), 30 cents.

An interpolation procedure for calculating atmospheric band absorptions from laboratory data, L. Droppleman, L. R. Megill, and R. F. Calfee, NBS Tech. Note 178 (June 3, 1963), 20 cents.

Relative power transmission characteristics of the ear and skull from hearing threshold data, E. L. Smith, Proc. Fourth Intern. Congr. on Acoustics, Part I, Paper H48 (Copenhagen, Denmark, Aug. 21-28, 1962).

Polymer research at the U.S. National Bureau of Standards, Part I, G. M. Kline, SPE J. 19, No. 3, 278-283 (Mar. 1963).

Polymer research at the U.S. National Bureau of Standards, Part 2, G. M. Kline, SPE J. 19, No. 4, 403-408 (Apr. 1963).

Radiation and the world we live in, L. S. Taylor, Radiology 80, No. 3, 358-368 (Mar. 1963).

Natural and synthetic rubbers, E. J. Parks and F. J. Linnig, Anal. Chem. 35, No. 5, 160R-178R (Apr. 1963).

Low-temperature thermometry, K. D. Timmerhaus, Book, Applied Cryogenic Engineering, Ed. R. W. Vance, Chapt. 4, 60-103 (John Wiley \& Sons, Inc., New York, N.Y., 1962).

On the isomerization of isobutyl radicals, J. R. MeNesby and W. M. Jackson, J. Chem. Phys. 38, No. 3, 692-693 (Feb. 1, 1963).

The electrophoretic mobility of asphaltenes in nitromethane, J. R. Wright and R. R. Minesinger, J. Colloid Sci. 18, 223-236 (Mar. 1963).
A suggestion for improving forecasts of geomagnetic storms, Y. Hakura and J. V. Lincoln, J. Geophys. Res. 68, No. 5, 1563-1564 (Mar. 1, 1963).

Observation of a $6300 \mathrm{~A}$ are in France, America, and Australia, F. E. Roach, D. Barbier, and R. A. Duncan, Ann. Geophys. 18, 390-391 (Oct.-Dec. 1962).

Maximum efficiency of a two-arm waveguide junction, R. W. Beatty, IEEE Trans. Microwave Theory and Tech. MT'T-11, 94 (Jan. 1963).

Applications of a semiconductor-surface-state charge-storage device, L. J. Swartzendruber, Solid-State Elec. 6, 59-61 (Pergamon Press, Inc., New York, N.Y., 1963).

Electric fields in the ionosphere and the excitation of the red lines of atomic oxygen, L. R. Megill, M. H. Rees, and L. K. Droppleman, Planetary Space Sci. 11, 45-56 (Jan. 1963).

Ten-kilocycle pound-type klystron stabilizer, H. E. Radford, Rev. Sci. Instr. 34, No. 3, 304-305 (Mar. 1963).

International geophysical calendar for 1963, A. H. Shapley and J. V. Lincoln, J. Geophys. Res. 68, No. 4, 1157-1159 (Feb. 15, 1963)

Kihara parameters and second virial coefficients for cryogenic fluids and their mixtures, J. M. Prausnitz and A. L. Myers, A. I. Ch. E. Journal 9, No. 1, 5-11 (Jan. 1963).

Standards and the microwave profession, J. M. Richardson, IRE Trans. Microwave Theory and Tech. MTT-10, No. 6, 413-415 (Nov. 1962).

Transparent rigid mount for vacuum stopcock, M. M. Anderson, Rev. Sci. Instr. 34, No. 2, 178 (Feb. 1963).

Delay time of polar-cap blackout and its relation to decay time of geomagnetic disturbance, C. S. Warwick, J. Geophys. Res. 68, No. 5, 1561-1562 (Mar. 1, 1963).

Electrolytic conductance of ammonium dihydrogen phosphate solutions in the saturation region, J. L. Torgesen and A. T. Horton, J. Phys. Chem. 67, 376-381 (1963).

Thermometry, low temperature, R. P. Hudson, Encyclopaedic Dictionary of Physics $\boldsymbol{7}, 323-325$ (1962).

Standards for the $70 \mathrm{~s}$, W. A. Wildhack, Ind. Res. 5, No. 3, 15-20 (Mar. 1963).

Effect of outdoor exposure on some properties of chromeretanned leather, T. J. Carter, J. Am. Leather Chemists Assoc. LVIII, No. 3, 155-160 (Mar. 1963).

Nuclear orientation, E. Ambler, Book, Methods of Experimental Physics 5, Sect. 2.4.2.3, 162-214 (Academic Press Inc., New York, N.Y., 1963).

APPA-TAPPI reference material program II. Effectiveness of a reference material in reducing the between-laboratory variability of TAPPI standard T $414 \mathrm{~m}-49$ for internal tearing resistance of paper, T. W. Lashof, Tappi 46, No. 3, 145-150 (Mar. 1963).

Some characteristics of a simple cryopump, L. O. Mullen and R. B. Jacobs, 1962 Trans. Ninth Natl. Vacuum Symp., Am. Vacuum Soc., pp. 220-226 (1963).

The ionosphere over Antaretica, W. R. Piggott and A. H. Shapley, Antarctic Research, Geophysical Mono. 7, pp. 111-126 (1962).

The personal side of a research project, A. T. MicPherson, J. Wash. Acad. Sci. 53, No. 3, 63-66 (Mar. 1963).

Indication limit, E. L. R. Corliss, Proc. Fourth Intern. Congr. Acoustics, Pt. I, Paper N22 (Copenhagen, Denmark, Aug. 21-28, 1962).

Nuclear resonance and the hyperfine field in dilute alloys of nickel in iron, R. L. Streever, L. H. Bennett, R. C. La Force, and G. F. Day, J. Appl. Phys. 34, No. 4, Pt. 2, 1050-1051 (Apr. 1963).

The history of Pt 27, E. Wichers Book, Temperature, Its Measurement and Control in Science and Industry 3, Pt. 1, 259-262 (Reinhold Publ. Corp., New York, N.Y., 1962).

Some causes of resonant frequency shifts in atomic beam machines. I. Shifts due to other frequencies of excitation, J. H. Shirley, J. Appl. Phys. 34, 783-788 (Apr. 1963). Some causes of resonant frequency shifts in atomic beam machines. II. The effect of slow frequency modulation on the Ramsey line shape, J. H. Shirley, J. Appl. Phys. 34, 789-791 (Apr. 1963).

On the dependence of absorption coefficients upon the area of the absorbent material, E. D. Daniel, J. Acoust. Soc. Am. 35, No. 4, 571-573 (Apr. 1963). 
The role of the International Union of Pure and Applied Chemistry, E. Wichers, J. Chem. Doc. 3, No. 7, 7-11 (1963).

New scale of nuclidic masses and atomic weights, E. Wichers, Nature 194, No. 4829, 621-624 (May 19, 1962).

Note on a subgroup of the modular group, M. Newman and J. R. Smart, Proc. Am. Math. Soc. 14, No. 1, 102-104 (Feb. 1963).

Calibration of photogrammetric lenses and cameras at the National Bureau of Standards, F. E. Washer, Pathogrammetric Eng. XXIX, No. 1, 113-119 (Jan. 1963).

The orthobaric densities of parahydrogen, derived heats of vaporization and critical constants, H. M. Roder, D. E. Diller, L. A. Weber, and R. D. Goodwin, Cryogenies 3, 16-22 (Mar. 1963).

Melting pressure equation for the hydrogens, R. D. Goodwin, Cryogenics 2, No. 6, 1-3 (Dec. 1962).

Isotopic fractionation of uranium in sandstone, J. N. Rosholt, W. R. Shields, and E. L. Garner, Science 139, 224-226 (Jan. 18, 1963).

The total electron content of the ionosphere at middle latitudes near the peak of the solar cycle, R. S. Lawrence, D. J. Posakony, O. K. Garriott, and S. C. Hall, J. Geophys. Res. 68, 1889-1898 (Apr. 1, 1963).

Present status of our knowledge of atomic transition probabilities, W. L. Wiese, Proc. Tenth Colloquium Spectroscopic Intern., pp. 37-56 (Univ. of Maryland, College Park, Md., 1962).

Rubber and rubber products, W. P. Tyler and M. Tryon, Book, Industrial and Natural Products and Noninstrumental Methods, 6th Ed., Standard Methods of Chemical Analysis IIB, Chapt. 43, 2146-2226 (D. Van Nostrand Co., Inc., New York, N.Y., 1963).

The formation and oxidation of high-area carbon films, V. R Deitz and E. F. McFarlane, Proc. Fifth Carbon Conference II, 219-232 (Pergamon Press, Inc., London, England, 1963).

Pure substance and mesaurement, E. Wichers, Mater. Res. Stds. 1, No. 4, 314-315 (Apr. 1961).

The specific heat at constant volume of parahydrogen at temperatures from 15 to $90^{\circ} \mathrm{K}$ and pressures to $340 \mathrm{~atm}$, B. A. Younglove and D. E. Diller, Cryogenies 2, No. 6, 1-5 (Dec. 1962).

Radiation detectors, L. Costrell, Science 139, No. 3558, 899 (Mar. 8, 1963).

Intercomparisons of laboratory test results, J. Mandel, ISA Proc. 17, 44.3.62-1 to $44.3 .62-5$ (1962).

Electron spin resonance of gamma-irradiated cellulose, R. E. Florin and L. A. Wall, J. Polymer Sci. 1, Pt. A, 1163-1173 (1963).

The speed of light, A. G. McNish, IRE Trans. Instr. I-11, Nos. $3 \& 4,138-148$ (Dec. 1962).

Fundamentals of measurement, A. G. MeNish, ElectroTechnol. 53, 113-128 (May 1963).

Optimum estimators of the parameters of negative exponential distributions from one or two order statistics, M. M. Siddiqui, Ann. Math. Stat. 34, 117-121 (Mar. 1963).

Building a simple transistor tester, G. F. Montgomery, Electronics 36, No. 16, 56 (Apr. 19, 1963).

Electron microscopy studies of the surfaces of magnetic recording media, F. Nesh and D. B. Ballard, IEEE Trans. Audio AU-11, No. 1, 15-18 (Jan. 2, 1963).

Two-stream plasma instability as a source of irregularities in the ionosphere, D. T. Farley, Jr., Phys. Rev. Letters 10, 279-282 (Apr. 1, 1963).

A magnetic amplifier for use with diode logic, E. W. Hogue, Proc. IEEE 1963 Intern. Conf. Nonlinear Magnetics No. T-149, 8.6-1 to 8.6-6 (Apr. 1963).

Kinetics of the acid-catalyzed hydrolysis of acetal in wateracetone solvents at 15,25 , and $35^{\circ}, \mathrm{R}$. K. Wolford, J. Phys. Chem. 6\%, 632-636 (1963).
Performance characteristics of split-type residential air-to-air heat pumps, J. C. Davis and P. R. Achenbach, Suppl. Bull. Inst. Intern. Refrigeration, pp. 1-7 (1961-1962).

Crystallographic changes with the substitution of aluminum for iron in dicalcium ferrite, D. K. Smith, Acta Cryst. 15, 1146-1152 (Jan. 1963).

Methods for the analysis of rubber and related products, M. Tryon and E. Horowitz, Handb. Analytical Chemistry, Sect. 13, pp. 233-256 (McGraw-Hill Book Co., Inc., New York, N.Y., 1963).

Pressure-density-temperature relations of freezing liquid parahydrogen to 350 atmospheres, R. D. Goodwin, Cryogenies 3, 12-15 (Mar. 1963).

Nuclear magnetic resonance in metal powders at low temperatures, R. J. Snodgrass and L. H. Bennett, J. Appl. Spectry. 17, No. 2, 53-54 (1963).

Millimeter wavelength resonant structures, R. W. Zimmerer, M. V. Anderson, G. L. Strine, and Y. Beers, IEEE Trans. Microwave Theory Tech. MTT-11, 142-149 (Miar. 1963).

Lunar point-to-point communication, L. E. Vogler, Book, Technology of Lunar Exploration, Progress in Astronautics and Aeronautics 9, 533-559 (Academic Press Inc., New York, N.Y., 1963).

Comment on "Parametric behavior of an ideal two-frequency varactor," G. F. Montgomery, Proc. IEEE 51, No. 3, 491 (Mar. 1963).

Distribution of latitude of red ares, E. Marovich and F. E. Roach, J. Geophys. Res. 68, No. 7, 1885-1888 (Apr. 1, 1963).

U.S. participation in international standardization, A. T. McPherson, ASTM Mater. Res. Stds. 3, No. 4, 310-311 (April 1963).

Experimental investigation of Fabry-Perot interferometer, R. W. Zimmerer, Proc. IEEE 51, 475-576 (Mar. 1963).

The following papers were published in the Proc. Intern. Conf. Ionosphere, London, 1962 (The Institute of Physics and The Physical Society, London, England):

A model of the atmosphere and the ionosphere in the $E$ and F1 regions, R. B. Norton, T. E. VanZandt, and J. S. Denison, pp. 26-34.

Doppler studies of the ionospheric effects of solar flares, $\mathrm{K}$. Davies, pp. 76-83.

Ionospheric variations during geomagnetic storms, S. Matsushita, pp. 120-127.

The Location of the irregularities responsible for ionospheric scintillation of a radio source, H. J. A. Chivers, pp. 258-266.

Equatorial spread- $F$ motions, W. Calvert, K. Davies, E. Stiltner, and J. T. Brown, pp. 316-322.

Evidence for field-aligned ionization irregularities between 400 and $1000 \mathrm{~km}$ above the earth's surface, W. Calvert, T. E. VanZandt, R. W. Knecht, and G. B. Goe, pp. 324-329.

Experimental observations and theoretical calculations leading to a model for the lower ionosphere, R. H. Doherty, pp. $428-434$.

Radio wave reflections at a continuously stratified plasma with electron collision frequency proportional to energy and arbitrary magnetic induction, J. R. Johler, pp. 436-445.

Very low frequency propagation in the earth-ionosphere waveguide of non-uniform width, J. R. Wait, pp. 446-451.

*Publications for which a price is indicated are available by purchase from the Superintendent of Documents, U.S. Government Printing Office, Washington D.C., 20402 (foreign postage, one-fourth additional). Reprints from outside journals and the NBS Journal of Research may often be obtained directly from the authors. 